\title{
LAG LENGTH SELECTION AND THE CONSTRUCTION OF UNIT ROOT TESTS WITH GOOD SIZE AND POWER
}

\author{
Serena $\mathrm{Ng}^{*} \quad$ Pierre Perron ${ }^{\dagger}$
}

September 2000

\begin{abstract}
It is widely known that when there are errors with a moving-average root close to -1 , a high order augmented autoregression is necessary for unit root tests to have good size, but that information criteria such as the $A I C$ and the $B I C$ tend to select a truncation lag $(k)$ that is very small. We consider a class of Modified Information Criteria $(M I C)$ with a penalty factor that is sample dependent. It takes into account the fact that the bias in the sum of the autoregressive coefficients is highly dependent on $k$ and adapts to the type of deterministic components present. We use a local asymptotic framework in which the moving-average root is local to -1 to document how the $M I C$ performs better in selecting appropriate values of $k$. In monte-carlo experiments, the $M I C$ is found to yield huge size improvements to the $D F G L S$ and the feasible point optimal $P_{T}$ test developed in Elliott, Rothenberg and Stock (1996). We also extend the $M$ tests developed in Perron and Ng (1996) to allow for $G L S$ detrending of the data. The $M I C$ along with $G L S$ detrended data yield a set of tests with desirable size and power properties.
\end{abstract}

Keywords: Integrated processes, truncation lag, $G L S$ detrending, information criteria. JEL Classification: C2,C3,C5

\footnotetext{
*Department of Economics, Boston College, Chestnut Hill, MA, 02467. Email: serena.ng@bc.edu

${ }^{\dagger}$ Department of Economics, Boston University, 270 Bay State Rd., Boston, MA, 02215. Email: perron@bu.edu This is a substantially revised version of a paper previously circulated as "Constructing Unit Root Tests with Good Size and Power". We thank three anonymous referees and the editor for comments on earlier drafts. The authors thank the Social Science and Humanities Research Council of Canada (SSHRC), the Natural Sciences and Engineering Research Council of Canada (NSERC) and the Fonds de la Formation de Chercheurs et l'Aide à la Recherche du Québec (FCAR) for financial support.

Correspondence: Serena Ng, Department of Economics, Boston College, Chestnut Hill, MA 02467.
} 


\section{Introduction}

Many unit root tests have been developed for testing the null hypothesis of a unit root against the alternative of stationarity. While the presence or absence of a unit root has important implications, many remain skeptical about the conclusions drawn from such tests. This concern is justifiable, as these tests generally suffer from two problems. First, many tests have low power when the root of the autoregressive polynomial is close to but less than unity (e.g., DeJong et al., 1992). Second, the majority of the tests suffer from severe size distortions when the moving-average polynomial of the first differenced series has a large negative root (e.g., Schwert, 1989, Perron and Ng, 1996). Although less severe, the problem also arises when there is a large negative autoregressive root in the residuals. The consequence is over-rejections of the unit root hypothesis. While few economic time series are found to have negative serial correlation of the autoregressive type, many do exhibit a large negative moving-average root. The inflation rate is an example, and omitted outliers have also been shown to induce a negative moving average root in the error process (e.g., Franses and Haldrup, 1994). It is therefore desirable to have powerful unit root tests that are robust to size distortions.

The implementation of unit root tests often necessitates the selection of an autoregressive truncation lag, say, $k$. This is required in the autoregression used to form the Said-Dickey-Fuller test. It is also required in constructing an autoregressive estimate of the spectral density at frequency zero. However, simulations have repeatedly shown a strong association between $k$ and the severity of size distortions and/or the extent of power loss (e.g., Ng and Perron, 1995, and Lopez, 1997). To date, there exists no operational procedure for selecting $k$ that yields robust and satisfactory results. The problem is that while a small $k$ is adequate for finite order autoregressive errors and $A R M A$ processes with small moving-average components, a large $k$ is generally necessary for noise functions with a moving-average root that is large. For the latter class of models, selection rules such as the Schwarz or Bayesian Information Criterion $(B I C)$ and the Akaike Information Criterion $(A I C)$ tend to select values of $k$ that are generally too small for unit root tests to have good sizes.

This paper sets out to resolve two problems. One is to enhance the power of several tests that have been shown to have small size distortions. More precisely, in Perron and Ng (1996), we analyzed a class of modified tests, originally proposed by Stock (1990), and showed that these tests are far more robust to size distortions than other unit root tests in the literature when the residuals have negative serial correlation. Exploiting the findings in Elliott et al. (1996) (hereafter ERS) and Dufour and King (1991) that local GLS detrending of the data yields substantial power gains, we apply the idea of $G L S$ detrending to the modified tests and show that non-negligible size and power gains can be made when used in conjunction with an autoregressive spectral density 
estimator at frequency zero provided the truncation lag is appropriately selected.

Our second task is to provide an improved procedure for choosing the truncation lag. We argue that the penalty of $k$ assigned to overfitting in information criteria such as the $A I C$ underestimates the cost of a low order model when the unit root process has a negative moving-average root and, hence, tends to select a lag length that is too small. We suggest a class of modified information criteria that takes better account of the cost of underfitting. The modified $A I C$ is shown to lead to substantial size improvements over standard information criteria in all the unit root tests considered. Taking the two steps together, GLS detrending along with the selection rule for $k$ provide procedures which allow for the construction of unit root tests with much improved size and power. Additionally, using GLS detrended data to estimate the spectral density at frequency zero is found to have favorable size and power implications.

The paper proceeds as follows. After setting up the preliminaries in Section 2, Section 3 considers the class of $M$ tests with $G L S$ detrending. Section 4 explains why information criteria will underestimate $k$ and Section 5 proposes an alternative penalty factor in the context of integrated data. Section 6 presents the properties of the modified information criteria using a local asymptotic framework and shows why the $A I C$ version is preferable. Simulation results are presented in Section 7 and the procedures are illustrated using inflation rates for the G7 countries in Section 8. Section 9 offers brief concluding remarks. Mathematical derivations are given in the Appendix.

\section{Preliminaries}

We assume that we have $T+1$ observations generated by (for $t=0, \ldots, T)$ :

$$
y_{t}=d_{t}+u_{t}, \quad u_{t}=\alpha u_{t-1}+v_{t}
$$

where $E\left(u_{0}^{2}\right)<\infty$ (even as $\left.T \rightarrow \infty\right), v_{t}=\delta(L) e_{t}=\sum_{j=0}^{\infty} \delta_{j} e_{t-j}$ with $\sum_{j=0}^{\infty} j\left|\delta_{j}\right|<\infty$ and $\left\{e_{t}\right\} \sim$ i.i.d. $\left(0, \sigma_{e}^{2}\right)$. The non-normalized spectral density at frequency zero of $v_{t}$ is given by $\sigma^{2}=\sigma_{e}^{2} \delta(1)^{2}$. Also, $T^{-1 / 2} \sum_{t=1}^{[s T]} v_{t} \Rightarrow \sigma W(s)$, where $\Rightarrow$ denotes weak convergence in distribution and $W(s)$ is a Wiener process defined on $C[0,1]$. In $(1), d_{t}=\psi^{\prime} z_{t}$, where $z_{t}$ is a set of deterministic components. We consider $d_{t}=\sum_{i=0}^{p} \psi_{i} t^{i}$, with special focus on $p=0,1$, though the analysis remains valid for more general cases ${ }^{1}$. Of interest is testing the null hypothesis $\alpha=1$ against $\alpha<1$. The $D F$ test due to Dickey and Fuller (1979) and Said and Dickey (1984) is the $t$ statistic for $\beta_{0}=0$ in the autoregression:

$$
\Delta y_{t}=d_{t}+\beta_{0} y_{t-1}+\sum_{j=1}^{k} \beta_{j} \Delta y_{t-j}+e_{t k} .
$$

\footnotetext{
${ }^{1}$ Most of the results presented here, in particular Theorem 1 below, hold with the less restrictive assumption that $\left\{e_{t}\right\}$ is a martingale difference sequence with respect to the sigma algebra $\mathcal{F}_{t}=\left\{e_{s} ; s \leq t\right\}$ with $\sigma_{e}^{2}=\lim _{T \rightarrow \infty} T^{-1} \sum_{t=1}^{T} E\left(e_{t}^{2} \mid \mathcal{F}_{t-1}\right)$.
} 
In Perron and $\mathrm{Ng}$ (1996), we analyzed the properties of three tests: $M Z_{\alpha}, M Z_{t}$ and $M S B$, collectively referred to as the $M$ tests. These are defined as (for the no deterministic term case):

$$
\begin{aligned}
M Z_{\alpha} & =\left(T^{-1} y_{T}^{2}-s_{A R}^{2}\right)\left(2 T^{-2} \sum_{t=1}^{T} y_{t-1}^{2}\right)^{-1}, \\
M S B & =\left(T^{-2} \sum_{t=1}^{T} y_{t-1}^{2} / s_{A R}^{2}\right)^{1 / 2},
\end{aligned}
$$

and $M Z_{t}=M Z_{\alpha} \times M S B$. All three tests are based on $s_{A R}^{2}$, an autoregressive estimate of the spectral density at frequency zero of $v_{t}$. Let $\hat{\beta}(1)=\sum_{i=1}^{k} \hat{\beta}_{i}, \hat{\sigma}_{k}^{2}=(T-k)^{-1} \sum_{t=k+1}^{T} \hat{e}_{t k}^{2}$, with $\hat{\beta}_{i}$ and $\left\{\hat{e}_{t k}\right\}$ obtained from (2) estimated by $O L S$. Then,

$$
s_{A R}^{2}=\hat{\sigma}_{k}^{2} /(1-\hat{\beta}(1))^{2} .
$$

The $M$ tests for $p=0$ and 1 can be obtained upon replacing $y_{t-1}$ and $y_{T}$ by the residuals obtained from least squares detrending. The $M Z_{\alpha}$ and $M Z_{t}$ tests can be viewed as modified versions of the Phillips (1987) and Phillips-Perron (1988) $Z_{\alpha}$ and $Z_{t}$ tests referred to as the $Z$ tests below (see Perron and $\mathrm{Ng}, 1998$ ). The $Z$ tests suffer from severe size distortions when $v_{t}$ has a negative moving-average root. When the root is close to minus one (e.g., -.8), the rejection rate can be as high as 100\% (see Schwert (1989)). Perron and Ng (1996) showed that the $M$ tests have dramatically smaller size distortions than most (if not all) unit root tests in the literature in cases of negative moving-average errors if the autoregressive spectral density estimator defined above is used in conjunction with a suitably chosen $k$. The three $M$ tests have similar properties and we use $M Z_{\alpha}$ for illustration.

\section{The Tests Under GLS Detrending}

To improve power, ERS proposed local to unity $G L S$ detrending of the data. For any series $\left\{x_{t}\right\}_{t=0}^{T}$, define $\left(x_{0}^{\bar{\alpha}}, x_{t}^{\bar{\alpha}}\right) \equiv\left(x_{0},(1-\bar{\alpha} L) x_{t}\right), t=1, \ldots, T$, for some chosen $\bar{\alpha}=1+\bar{c} / T$. The $G L S$ detrended series is defined as

$$
\widetilde{y}_{t} \equiv y_{t}-\hat{\psi}^{\prime} z_{t}
$$

where $\hat{\psi}$ minimizes $S(\bar{\alpha}, \psi)=\left(y^{\bar{\alpha}}-\psi^{\prime} z^{\bar{\alpha}}\right)^{\prime}\left(y^{\bar{\alpha}}-\psi^{\prime} z^{\bar{\alpha}}\right)$. If $v_{t}$ is $i . i . d$. normal, the point optimal test of the null hypothesis $\alpha=1$ against the alternative hypothesis $\alpha=\bar{\alpha}$ is the likelihood ratio statistic, $L=S(\bar{\alpha})-S(1)$, where $S(\bar{\alpha})=\min _{\psi} S(\bar{\alpha}, \psi)$. ERS considered a feasible point optimal test which takes into account that $v_{t}$ may be serially correlated. The statistic is

$$
P_{T}=[S(\bar{\alpha})-\bar{\alpha} S(1)] / s_{A R}^{2}
$$


where $s_{A R}^{2}$ is defined as in (5). The value of $\bar{c}$ is chosen such that the asymptotic local power function of the test is tangent to the power envelope at $50 \%$ power. For $p=0$ this is -7.0 and for $p=1$, it is -13.5 . The $P_{T}$ test did not perform well in simulations when $k$ was selected by the $B I C$. Large size distortions were recorded. ERS then recommended the $D F^{G L S}$ statistic (whose local asymptotic power function also lies close to the Gaussian local power envelope) as the t-statistic for testing $\beta_{0}=0$ from the following regression estimated by $O L S$ :

$$
\Delta \widetilde{y}_{t}=\beta_{0} \widetilde{y}_{t-1}+\sum_{j=1}^{k} \beta_{j} \Delta \widetilde{y}_{t-j}+e_{t k} .
$$

\subsection{The $M^{G L S}$ tests}

While the power gains of the $D F^{G L S}$ from using $G L S$ detrended data are impressive, simulations also show that the test exhibits strong size distortions when $v_{t}$ is an $M A(1)$ with a negative coefficient. For $T=100$, ERS report rejection rates of 30 and $50 \%$ for $p=0$ and 1 , respectively, when the nominal rate is $5 \%$. An explanation is provided in Section 4. Size distortions, however, are less of an issue with the $M$ tests in theory as we showed in Perron and $\mathrm{Ng}$ (1996). In practice, it does require us to have a way to find the appropriate $k$. Suppose for the moment that this lag length selection problem is solved. Since the power gains from the $D F^{G L S}$ over the $D F$ comes from the use of $G L S$ detrended data, it is natural to consider the $M$ tests under $G L S$ detrending. We now state the asymptotic properties of the $M^{G L S}$ tests.

Theorem 1 Let $y_{t}$ be generated as in (1) with $\alpha=1+c / T$ and consider data obtained from local GLS detrending at $\bar{\alpha}=1+\bar{c} / T$. Also, let $s_{A R}^{2}$ be a consistent estimate of $\sigma^{2}$. If $p=0$, $M Z_{\alpha}^{G L S} \Rightarrow\left(\int_{0}^{1} J_{c}(r) d W(r)\right)\left(\int_{0}^{1} J_{c}(r)^{2} d r\right)^{-1}$ and $M S B^{G L S} \Rightarrow\left(\int_{0}^{1} J_{c}(r)^{2} d r\right)^{1 / 2}$. If $p=1, M Z_{\alpha}^{G L S} \Rightarrow$ $\left.0.5\left(V_{c, \bar{c}}(1)^{2}-1\right)\right)\left(\int_{0}^{1} V_{c, \bar{c}}(r)^{2} d r\right)^{-1}$ and $M S B^{G L S} \Rightarrow\left(\int_{0}^{1} V_{c, \bar{c}}(r)^{2} d r\right)^{1 / 2}$, where $J_{c}(r)$ is an OrnsteinUhlenbeck process defined by $d J_{c}(r)=c J_{c}(r) d r+d W(r)$ with $J_{c}(0)=0$, and $V_{c, \bar{c}}(r)=J_{c}(r)-$ $r\left[\lambda J_{c}(1)+3(1-\lambda) \int_{0}^{1} s J_{c}(s) d s\right], \lambda=(1-\bar{c}) /\left(1-\bar{c}+\bar{c}^{2} / 3\right)$. Results for $M Z_{t}^{G L S}$ can be obtained in view of the fact that $M Z_{t}^{G L S}=M Z_{\alpha}^{G L S} \times M S B^{G L S}$.

The proof is based on standard results for sample moments of $G L S$ detrended data and therefore omitted. The $M Z_{\alpha}^{G L S}, M Z_{t}^{G L S}$ and $M S B^{G L S}$ have similar size and power properties and will be referred to as the $M^{G L S}$ tests. We have computed, via simulations, the local asymptotic power functions of the $M$ tests with $O L S$ and GLS detrending. These showed the $M^{G L S}$ tests to be uniformly more powerful than the $M$ tests and also to have local asymptotic power functions that are indistinguishable from those of the $D F^{G L S}$ and the Gaussian asymptotic local power envelope. A graph is therefore not presented. The important point is that the $M^{G L S}$ tests, like the $D F^{G L S}$, 
achieve local asymptotic power that is approximately the same as the feasible point-optimal test. We also consider two modified feasible point optimal tests and derived their limiting distributions:

$$
\begin{aligned}
& p=0: \quad M P_{T}^{G L S}=\left[\bar{c}^{2} T^{-2} \sum_{t=1}^{T} \widetilde{y}_{t-1}^{2}-\bar{c} T^{-1} \widetilde{y}_{T}^{2}\right] / s_{A R}^{2} \Rightarrow \bar{c}^{2} \int_{0}^{1} J_{c}(r)^{2} d r-\bar{c} J_{c}(1)^{2}, \\
& p=1: \quad M P_{T}^{G L S}=\left[\bar{c}^{2} T^{-2} \sum_{t=1}^{T} \widetilde{y}_{t-1}^{2}+(1-\bar{c}) T^{-1} \widetilde{y}_{T}^{2}\right] / s_{A R}^{2} \Rightarrow \bar{c}^{2} \int_{0}^{1} V_{c, \bar{c}}(r)^{2} d r+(1-\bar{c}) V_{c, \bar{c}}(1)^{2} .
\end{aligned}
$$

These tests are based on the same motivation that leads to the definition of the $M$ tests in Stock (1990), namely, to provide functionals of sample moments that have the same asymptotic distributions as well known unit root tests. The $M P_{T}$ is important because its limiting distribution coincide with that of the feasible point optimal test considered in ERS.

Critical values for the limiting distributions of the $M^{G L S}$ tests were obtained by numerical simulations which approximate the Wiener process $W(r)$ by partial sums of $N(0,1)$ random variables with 5,000 steps and 20,000 simulations. These are summarized in Table 1.

\subsection{The Construction of the Autoregressive Spectral Density Estimator and the $\bar{M}^{G L S}$}

All that is required for Theorem 1 and the results of ERS to hold is that $s_{A R}^{2}$ converges to $\sigma^{2}$ under the null hypothesis of a unit root, and for the tests to be consistent, that $T s_{A R}^{2}$ diverges under the alternative of stationarity. The attractiveness of $G L S$ detrending is that it estimates the deterministic function with more precision and leads to reduced bias in $\hat{\beta}_{i}$. Since this should lead to a more precise estimate of $\hat{\beta}(1)$, we also consider estimating $s_{A R}^{2}$ using the autoregression (8) based on $G L S$ detrended data. An additional advantage is that this estimator is invariant to the slope of the trend function ${ }^{2}$. Experimentation with different values of $\bar{c}$ when constructing the autoregressive spectral density gave similar results. Hence, $\bar{c}=-7.0$ and -13.5 will continue to be used. The tests so constructed are denoted $\bar{Z}^{G L S}$ and $\bar{M}^{G L S}$. These are to be distinguished from the $Z^{G L S}$ and $M^{G L S}$ statistics which use least squares detrended data to estimate $s_{A R}^{2}$.

\section{The Size Issue, the Selection of $k$, and Information Criteria}

To see the influence of the lag order $k$, we consider the finite sample size of the tests $\bar{M} Z_{\alpha}^{G L S}$, $D F^{G L S}$ and $\bar{Z}_{\alpha}^{G L S}$. The setup, which will be used throughout, is to consider data generated by (1) with $\alpha=1$ and $v_{t}$ is either an $M A(1)$, i.e. $v_{t}=e_{t}+\theta e_{t-1}$, or an $A R(1)$, i.e. $v_{t}=\rho v_{t-1}+e_{t}$, with $e_{t} \sim$ i.i.d. $N(0,1)$. The errors are generated from the $r n d n()$ function in Gauss with 999 as the

\footnotetext{
${ }^{2}$ When $p=1$, Perron and Ng (1996), Stock (1990) and ERS estimate equation (2) with only a constant by ordinary least-squares. This still ensures a consistent estimate under the null hypothesis but the omission of the trend term implies a non-invariance with respect to the slope of the trend function in finite samples under the alternative hypothesis. This non-invariance is such that the tests loose power as the slope increases.
} 
seed, and 5,000 replications are used. We use the nominal size of 5\% as the benchmark. Reported in Table 2 are the sizes of the tests for $p=0,1$ at selected values of $\theta$ and $\rho$. We report results for $T=100$ and 250 to highlight the fact that the size issue in the negative moving average case is not a small sample problem.

Several features of the results for $M A$ errors are of note. First, for a $\theta$ of the same absolute value, a negative $\theta$ always requires a larger lag to obtain a more accurate size. Second, for a positive $\theta$, the size of the tests are significantly better when $k$ is even. Third, the larger the number of deterministic terms, the more distant are the exact from the nominal sizes. Fourth, there is always a value of $k$ that will yield an $\bar{M} Z_{\alpha}^{G L S}$ with a reasonably accurate size when $\theta=-0.80$. For example, for $p=0$ when $T=100$ and 250, exact sizes of .077 and .055 , respectively, are achievable with lags of 9 and 8 . In contrast, there does not exist a $k$ that will result in an acceptable size for $\bar{Z}_{\alpha}^{G L S}$, and increasing the sample size will not reduce the size distortions adequately. For the sample sizes reported, the exact sizes for $\bar{Z}_{\alpha}^{G L S}$ are well above 0.5 at $\theta=-.8$, and size distortions are non-negligible even when $\theta=-0.5$. Fifth, the $D F^{G L S}$ also exhibits a dependence on $k$. At $T=100$, the size is .104 at $k=10$, but is .624 when $k=2$.

For $A R$ errors, size discrepancies between $\bar{Z}_{\alpha}^{G L S}$ and $\bar{M}_{\alpha}^{G L S}$ also exist when $\rho$ is large and negative, albeit not as dramatic as in the $M A$ case. The results reveal that the $\bar{M} Z_{\alpha}^{G L S}$ is inferior to the $D F^{G L S}$ when $p=1$, the sample size is small, and $|\rho|$ is large. For large negative $A R$ coefficients, the $\bar{M} Z_{\alpha}^{G L S}$ has a more accurate size at $k$ greater than 1. For large positive $A R$ coefficients, the $\bar{M} Z_{\alpha}^{G L S}$ is oversized for small $T$, though the size improves as $T$ increases.

Clearly, the choice of $k$ is crucial especially in the case of negative moving average errors. Various practical solutions have been used against this problem. In ERS, the $B I C$ is used to select $k$ but they set the lower bound to be 3 , because even larger size distortions would have resulted if zero was the lower bound. An alternative method is the sequential $t$ test for the significance of the last lag considered in $\mathrm{Ng}$ and Perron (1995). It has the ability to yield higher $k$ s than the $B I C$ when there are negative moving-average errors and reduce size distortions. But, the sequential test tends to overparameterize in other cases. This, as does ERS's implementation of the $B I C$, leads to less efficient estimates and subsequently to power losses. Neither approach is fully satisfactory.

The $A I C$ and the $B I C$ belong to the class of information based rules (hereafter, $I C$ ) where the chosen value of $k$ is $k_{i c}=\arg \min _{k \in\left[0, \ldots, k_{\max }\right]} I C(k)$ where ${ }^{3}$

$$
I C(k)=\ln \left(\hat{\sigma}_{k}^{2}\right)+(k+p+1) C_{T} /\left(T-k_{\max }\right),
$$

where $\hat{\sigma}_{k}^{2}=\left(T-k_{\max }\right)^{-1} \sum_{t=k+1}^{T} \hat{e}_{t k}^{2}, C_{T} / T \rightarrow 0$ as $T \rightarrow \infty$, and $C_{T}>0$. The various criteria differ

\footnotetext{
${ }^{3}$ See Ng and Perron (2000) for an analysis pertaining to the adequacy of the particular formulation of the information criterion stated.
} 
in $C_{T}$, the weight applied to overfitting, but all use $k$ as the penalty to overfitting. We argue below that, with integrated data, this penalty may be a poor approximation to the cost of underfitting.

\section{An Alternative Penalty for Integrated Data.}

To motivate the main idea, we consider the derivation of the $A I C$ for data generated by a finite order $A R\left(k_{0}\right)$ with normal errors and a unit root (i.e., the DGP is (2) with $k=k_{0}, \beta_{0}=0$ and $\left.e_{t k}=e_{t} \sim i . i . d . N(0,1)\right)$. The family of parametric models fitted is given by (8). For notation, we let $\beta^{0}(k)=\left(0, \beta_{1}, \ldots, \beta_{k}\right)^{\prime}, \hat{\beta}(k)=\left(\hat{\beta}_{0}, \hat{\beta}_{1}, \ldots, \hat{\beta}_{k}\right)^{\prime}, \beta_{-0}^{0}(k)=\left(\beta_{1}, \ldots, \beta_{k}\right)^{\prime}$ and $\hat{\beta}_{-0}(k)=\left(\hat{\beta}_{1}, \ldots, \hat{\beta}_{k}\right)^{\prime}$. In what follows, we adopt the treatment of Gourieroux and Monfort (1995, pp. 307-309). The goal is to select an autoregressive order between 0 and some upper bound $k_{\max }$. We assume this upper bound to satisfy $k_{\max } / T \rightarrow 0$ as $T \rightarrow \infty$. Let $f\left(\Delta y \mid \beta^{0}(k)\right)$ be the likelihood function of the data $\left(\Delta y_{k_{\max }+1}, \ldots, \Delta y_{T}\right)$ conditional on the initial observations $\left(y_{0}, \ldots, y_{k_{\max }}\right)$. This ensures that each competing model is evaluated with the same number of effective observations, namely $T-k_{\max }$. The Kullback distance between the true probability distribution and the estimated parametric model is $Q=E_{0}\left[\ln \left(f\left(\Delta y \mid \beta^{0}(k)\right)\right)-\ln (f(\Delta y \mid \hat{\beta}(k)))\right]$ with sample analog:

$$
\widetilde{Q}=\left(T-k_{\max }\right)^{-1} \sum_{t=k_{\max }+1}^{T} \ln \left(f\left(\Delta y_{t} \mid \beta^{0}(k)\right)\right)-\left(T-k_{\max }\right)^{-1} \sum_{t=k_{\max }+1}^{T} \ln \left(f\left(\Delta y_{t} \mid \hat{\beta}(k)\right)\right) .
$$

As discussed in Gourieroux-Monfort (1995), Akaike's suggestion was to find a $Q^{*}$ such that $\lim _{T \rightarrow \infty}$ $E\left[T\left(Q-Q^{*}\right)\right]=0$ so that $Q^{*}$ is unbiased for $Q$ to order $T^{-1}$. Let $X_{t}=\left(\widetilde{y}_{t-1}, Z_{t}\right)$ with $Z_{t}=$ $\left(\Delta \widetilde{y}_{t-1}, \ldots, \Delta \widetilde{y}_{t-k}\right)$ and

$$
\Phi_{T}(k)=\left(1 / \hat{\sigma}_{k}^{2}\right)\left(\hat{\beta}(k)-\beta^{0}(k)\right)^{\prime} \sum_{t=k_{\max }+1}^{T} X_{t} X_{t}^{\prime}\left(\hat{\beta}(k)-\beta^{0}(k)\right),
$$

where $\hat{\sigma}_{k}^{2}=\left(T-k_{\max }\right)^{-1} \sum_{t=k_{\max }+1}^{T} \hat{e}_{t k}^{2}$. Using Taylor series expansions, we have $T Q=\Phi_{T}(k) / 2+$ $o_{p}(1)$ and $T \widetilde{Q}=-\Phi_{T}(k) / 2+o_{p}(1)$. Since $T(Q-\widetilde{Q})=\Phi_{T}(k)+o_{p}(1), \lim _{T \rightarrow \infty} E\left[T\left(Q-Q^{*}\right)\right]=$ 0 if $Q^{*}=\widetilde{Q}+\Phi_{T}(k)$ and the remainder term is uniformly bounded. Now consider the term $\Phi_{T}(k)$ in the context of integrated data. Given the asymptotic block diagonality of the matrix $D_{T} \sum_{t=k_{\max }+1}^{T} X_{t} X_{t}^{\prime} D_{T}$ with $D_{T}=\operatorname{diag}\left(\left(T-k_{\max }\right)^{-1},\left(T-k_{\max }\right)^{-1 / 2}, \ldots,\left(T-k_{\max }\right)^{-1 / 2}\right)$, we have

$$
\begin{aligned}
\Phi_{T}(k) & =\left(\frac{1}{\hat{\sigma}_{k}^{2}}\right) \hat{\beta}_{0}^{2} \sum_{t=k_{\max }+1}^{T} \widetilde{y}_{t-1}^{2}+\left(\frac{1}{\hat{\sigma}_{k}^{2}}\right)\left(\hat{\beta}_{-0}(k)-\beta_{-0}^{0}(k)\right)^{\prime} \sum_{t=k_{\max }+1}^{T} Z_{t} Z_{t}^{\prime}\left(\hat{\beta}_{-0}(k)-\beta_{-0}^{0}(k)\right)+o_{p}(1) \\
& =\left(1 / \hat{\sigma}_{k}^{2}\right) \hat{\beta}_{0}^{2} \sum_{t=k_{\max }+1}^{T} \widetilde{y}_{t-1}^{2}+\chi_{k}^{2}+o_{p}(1)
\end{aligned}
$$

where $\chi_{k}^{2}$ is a chi-square random variable with $k$ degrees of freedom that is asymptotically independent of the first term. Hence, assuming the remainder term is uniformly integrable, a $Q^{*}$ that 
will satisfy $\lim _{T \rightarrow \infty} E\left[T\left(Q-Q^{*}\right)\right]=0$ is

$$
\begin{aligned}
Q^{*}= & \left(T-k_{\max }\right)^{-1} \sum_{t=k_{\max }+1}^{T} \ln \left(f\left(\Delta y_{t} \mid \beta^{0}(k)\right)\right)-\left(T-k_{\max }\right)^{-1} \sum_{t=k_{\max }+1}^{T} \ln \left(f\left(\Delta y_{t} \mid \hat{\beta}(k)\right)\right) \\
& +\left[\left(1 / \hat{\sigma}_{k}^{2}\right) \hat{\beta}_{0}^{2} \sum_{t=k_{\max }+1}^{T} \widetilde{y}_{t-1}^{2}+k\right] .
\end{aligned}
$$

Since the first term is common to all models and the second term is proportional to - $((T-$ $\left.\left.k_{\max }\right) / 2\right) \ln \left(\hat{\sigma}_{k}^{2}\right)$ under normality, minimizing $Q^{*}$ is equivalent to minimizing

$$
M A I C(k)=\ln \left(\hat{\sigma}_{k}^{2}\right)+\frac{2\left(\tau_{T}(k)+k\right)}{T-k_{\max }},
$$

where $\tau_{T}(k)=\left(\hat{\sigma}_{k}^{2}\right)^{-1} \hat{\beta}_{0}^{2} \sum_{t=k_{\max }+1}^{T} \widetilde{y}_{t-1}^{2}$ and $\hat{\sigma}_{k}^{2}=\left(T-k_{\max }\right)^{-1} \sum_{t=k_{\max }+1}^{T} \hat{e}_{t k}^{2}$. Note that the same result holds in the general case where the data are generated by (1). The important step is the relation given by (11) which can be shown to hold in the general case, in the sense that the remainder term is $o_{p}(1)$ uniformly in $k$ for $0<k \leq K_{T}$, where $K_{T}=o\left(T^{1 / 3}\right)$. This follows using the results of Berk (1974) and Said and Dickey (1984) provided $k \rightarrow \infty, k^{3} / T \rightarrow 0$ and $T^{-1 / 2} \sum_{j=k+1}^{\infty}\left|\beta_{i}\right| \rightarrow 0$.

As a natural generalization of the $M A I C(k)$, we propose a class of Modified Information Criteria ( $M I C)$ which selects $k$ as $k_{m i c}=\arg \min _{k} M I C(k)$ where

$$
M I C(k)=\ln \left(\hat{\sigma}_{k}^{2}\right)+\frac{C_{T}\left(\tau_{T}(k)+k\right)}{T-k_{\max }},
$$

with $C_{T}>0$ and $C_{T} / T \rightarrow 0$ as $T \rightarrow \infty$. The $M B I C$ obtains with $C_{T}=\ln \left(T-k_{\max }\right)$.

There are two important elements that distinguish (13) from standard information criteria. The first is the imposition of the null hypothesis $\beta_{0}=0$. This idea of imposing the null hypothesis in model selection when the ultimate objective is hypothesis testing appears new and may have implications beyond the unit root issue ${ }^{4}$. This is an interesting avenue of research, but is beyond the scope of this paper. The second element is that we retain a term in the penalty function that is stochastic. Now, as $T \rightarrow \infty,\left(1 / \hat{\sigma}_{k}^{2}\right) \hat{\beta}_{0}^{2} \sum_{t=k_{\max }+1}^{T} \widetilde{y}_{t-1}^{2} \Rightarrow\left(\sigma^{2} / \sigma_{e}^{2}\right)\left[\widetilde{W}(1)^{2}-1\right]^{2} /\left[2 \int_{0}^{1} \widetilde{W}(r)^{2} d r\right] \equiv$ $\tau(\widetilde{W})$, say, where $\widetilde{W}$ is a detrended Wiener process, whose exact form depends on the deterministic components and the method of detrending. In principle, we could use the mean of $\tau(\widetilde{W})$, which is independent of $k$, instead of $\tau_{T}(k)$, to construct $Q^{*}$ and the objective function would then reduce to the standard $A I C$. A central theme of our argument for retaining $\tau_{T}(k)$ is that, unless both $T$ and $k$ are very large, it varies substantially with $k$, especially when a negative $M A$ component is present. In a sense, the imposition of the null hypothesis, $\beta_{0}=0$, allows us to avoid using asymptotic expected values to approximate the penalty factor. We can therefore hope that $\tau_{T}(k)$ will better capture the relevant cost of selecting different orders in finite samples since it depends

\footnotetext{
${ }^{4}$ We thank a referee for bringing this feature to our attention.
} 
not only on $k$, but also on the nature of the deterministic components and the detrending procedure. Hence, the penalty term will also depend on these factors.

To get an idea of the dependence of $\tau_{T}(k)$ on $k$, Figure 1 graphs the average over 5,000 replications of $\tau_{T}(k)$ against $k$ for $p=0,1$ using the DGP $\Delta y_{t}=e_{t}+\theta e_{t-1}$ with $e_{t} \sim$ i.i.d. $N(0,1)$. For positive $\theta, \tau_{T}(k)$ is indeed fairly invariant to $k$ (as is the case for $A R$ processes, not reported). But, there is a clear inverse and non-linear relation between $\tau_{T}(k)$ and $k$ for negative values of $\theta$. This relation exists even when $\theta$ is -.5 for both $p=0$ and $p=1$. The basic reason for the dependence of $\tau_{T}(k)$ on $k$ is that the bias in the sum of the estimated autoregressive coefficients (i.e., $\hat{\beta}_{0}$ ) is very high for small values of $k$. This suggests that $\tau_{T}(k)$ will play a role in determining the appropriate $k$ when there are negative moving-average errors. To make this claim precise, the next subsection uses a local asymptotic framework to analyze the properties of $\tau_{T}(k)$ and the $M I C$.

\section{Local Asymptotic Properties.}

As discussed above, $\tau_{T}(k)$ will likely influence the choice of $k$ when there is a large negative moving-average root, a situation for which standard unit root tests exhibit severe size distortions. To understand the issue of lag length selection in this setting, a useful device is an asymptotic framework in which the moving average coefficient is parameterized as local to -1 .

$$
\begin{aligned}
y_{t}-\alpha_{T} y_{t-1} & =e_{t}+\theta_{T} e_{t-1}, \\
\alpha_{T}=1+c / T, \quad \theta_{T} & =-1+\delta / \sqrt{T},
\end{aligned}
$$

$e_{t} \sim$ i.i.d. $\left(0, \sigma_{e}^{2}\right), y_{0}=e_{0}=0, \delta>0$, and $c<0$. As $T$ increases to infinity, $\alpha_{T} \rightarrow 1, \theta_{T} \rightarrow-1$, and $y_{t}$ becomes a white noise process. However, $y_{t}$ is a nearly integrated nearly white noise process in finite samples. Nabeya and Perron (1994) used this asymptotic framework to derive approximations to the finite sample properties of the least-squares estimator in a first-order autoregressive model with negative moving-average errors and showed these approximations not only to be superior than those obtained from standard asymptotic distributions but also to be quite accurate even for small sample sizes. Perron and Ng (1996) used the same setup to analyze the size and power of various unit root tests based upon augmented autoregressions. Our aim here is to use the same device to first obtain useful qualitative features about the relative properties of the $I C$ and $M I C$, and second, to guide us to an appropriate choice of the penalty weight $C_{T}$.

\subsection{Local Asymptotic Properties of $\tau_{T}(k)$.}

The following Theorem (proved in the Appendix) summarizes the general properties of $\tau_{T}(k)$ in this local asymptotic framework. 
Theorem 2 Let $y_{t}$ be generated by (14), $Z_{1}=\left(1+\delta^{2} \int_{0}^{1} J_{c}(r)^{2} d r\right) /\left[\delta^{2} \int_{0}^{1} J_{c}(r)^{2} d r\right]^{2}$ when $p=0$, and $Z_{1}=\left(1+\delta^{2} \int_{0}^{1} V_{c, \bar{c}}(r)^{2} d r\right) /\left[\delta^{2} \int_{0}^{1} V_{c, \bar{c}}(r)^{2} d r\right]^{2}$ when $p=1$. Let $k \rightarrow \infty, K_{1 T}=o\left(T^{1 / 2}\right)$, $T^{1 / 2} / K_{2 T}=o_{p}(1)$ and $K_{3 T}=o(T)$. Then, 1) uniformly in $0<k \leq K_{1 T}$ : $\left(k^{2} / T\right) \tau_{T}(k) \Rightarrow Z_{1}$; 2) if $k / T^{1 / 2} \rightarrow \kappa, \tau_{T}(k) \Rightarrow \vartheta(\kappa)^{2} Z_{2}$, where $E\left(Z_{2}\right)=E\left(Z_{1}\right)$ and $\vartheta(\kappa)=2 \delta \exp (-2 \kappa \delta) /(1-\exp (-2 \kappa \delta))$; and 3) uniformly in $K_{2 T} \leq k \leq K_{3 T}, \tau_{T}(k) \Rightarrow Z_{3}$, where $Z_{3}$ is $O_{p}(1)$.

Theorem 2 shows that the local limit of $\tau_{T}(k)$ is unbounded unless $k$ increases at least at rate $T^{1 / 2}$. Since $\tau_{T}(0)$ diverges at rate $T$ (see Nabeya and Perron, 1994), $\tau_{T}(k)$ diverges at rate $T / k^{2}$ if $k^{2} / T \rightarrow 0$, the cost to overfitting is not linear in $k$ as is assumed for the $I C$, and Figure 1 bears this out. Theorem 2 also highlights the fact that $\tau_{T}(k)$ will depend, via $Z_{1}$, on the nature of the deterministic components and the method of detrending.

Given Theorem 2 and the fact that $\hat{\sigma}_{k}^{2}$ is consistent for $\sigma_{e}^{2}$ irrespective of the rate of increase of $k$ (shown in the Appendix), it can be shown that, in this local asymptotic framework, the limiting distribution of the $D F$ test diverges to $-\infty$ unless $k$ grows at least at rate $T^{1 / 2} .^{5}$ Hence, the unit root will always be rejected if standard critical values are used. This explains why the $D F$ tends to be oversized when $k$ is small. In Perron and $\mathrm{Ng}(1996,1998)$, we showed that $k^{2} s_{A R}^{2}=O_{p}(1)$ but we also need $T s_{A R}^{2}$ to be $O_{p}(1)$ for the $M$ tests to be bounded under the null hypothesis in this local framework. But $k^{2} s_{A R}^{2}$ and $T s_{A R}^{2}$ can both be bounded only if $k / T^{1 / 2} \rightarrow \kappa$ for some $0<\kappa<\infty$. In the next section, we will consider model selection procedures with this requirement in mind.

\subsection{Local Asymptotic Properties of the $M I C$ and $I C$}

In the standard asymptotic framework, with data generated by $(1), \tau_{T}(k)$ is invariant to $k$ for $T$ and $k$ large. It follows that $k_{m i c}$ grows at a logarithmic rate from Hannan and Deistler (1988). We now consider the properties of $k$ chosen by the $M I C$ and the $I C$ in the local asymptotic framework with the additional technical requirement that $C_{T}^{2} / T \rightarrow 0$. Using Theorem 2 and an expansion for $\ln \left(\hat{\sigma}_{k}^{2}\right)$ derived in the appendix, we have the following expansions for the $M I C$ and $I C$.

Lemma 1 Let $y_{t}$ be generated by (14) and $K_{1 T}, K_{2 T}$ and $K_{3 T}$ as defined in Theorem 2; then 1) uniformly in $0<k \leq K_{1 T}$ :

$$
M I C(k)=\ln \left(\sigma_{e}^{2}\right)+\frac{1}{k}+\frac{C_{T}}{k^{2}} Z_{1}+C_{T} \frac{k}{T}+O_{p}\left(T^{-1 / 2}\right) ;
$$

2) for $k / T^{1 / 2} \rightarrow \kappa$, with $\vartheta(\kappa)$ defined in Theorem 2 :

$$
M I C(k)=\ln \left(\sigma_{e}^{2}\right)+\frac{\vartheta(\kappa)}{\sqrt{T}}+\frac{C_{T}}{T} \vartheta(\kappa)^{2} Z_{2}+C_{T} \frac{\kappa}{\sqrt{T}}+O_{p}\left(T^{-1 / 2}\right)
$$

\footnotetext{
${ }^{5}$ This is a more general result than Pantula (1991) who used a different local asymptotic framework for modeling the negative moving average component in unit root tests. Pantula's result applies only when $k=O_{p}\left(T^{1 / 4}\right)$.
} 
3) uniformly in $K_{2 T} \leq k \leq K_{3 T}$ :

$$
M I C(k)=\ln \left(\sigma_{e}^{2}\right)+\frac{2 \delta}{\sqrt{T} \exp (2 k \delta / \sqrt{T})}+\frac{C_{T}}{T} Z_{3}+C_{T} \frac{k}{T}+O_{p}\left(T^{-1 / 2}\right) ;
$$

The expansions for the $I C(k)$ are the same except that the terms involving the $Z$ 's are absent.

The objects of ultimate interest are the values $k_{i c}$ and $k_{m i c}$ chosen by the $I C$ and the $M I C$, respectively, over all possible values of $k$.

Theorem 3 Let $y_{t}$ be generated by (14) and consider the rate of increase of $k$ as $T \rightarrow \infty$. Define:

$$
\begin{aligned}
F_{M I C(1)}(\kappa) & =\frac{-4 \delta^{2} \exp (-2 \kappa \delta)}{\sqrt{T}[1-\exp (-2 \kappa \delta)]^{2}}+C_{T}\left(\frac{-16 \delta^{3}}{T} \frac{\exp (-2 \kappa \delta)^{2} Z_{2}}{[1-\exp (-2 \kappa \delta)]^{3}}+\frac{1}{\sqrt{T}}\right) \\
F_{M I C(2)}(\kappa) & =-\frac{\sqrt{C_{T} / T}}{\kappa^{2}}+\sqrt{C_{T} / T}-\frac{2 C_{T}^{2}}{\kappa^{3} T} Z_{1} \\
F_{I C}(\kappa) & =\frac{-4 \delta^{2} \exp (-2 \kappa \delta)}{[1-\exp (-2 \kappa \delta)]^{2}}+C_{T} .
\end{aligned}
$$

When $C_{T}$ is fixed, $k_{\text {mic }} /\left(\kappa_{\text {mic }(1)} T^{1 / 2}\right) \rightarrow 1$ where $\kappa_{\text {mic }(1)}$ solves $F_{M I C(1)}(\kappa)=0$, and $k_{i c} /\left(\kappa_{i c} T^{1 / 2}\right) \rightarrow$ 1 , where $\kappa_{i c}$ solves $F_{I C}(\kappa)=0$. When $C_{T} \rightarrow \infty, k_{i c} / \sqrt{T / C_{T}} \rightarrow 1$. When $C_{T} \rightarrow \infty$ and $C_{T}^{3} / T \rightarrow d$ $(\geq 0), k_{m i c} /\left(\kappa_{m i c}(2) \sqrt{T / C_{T}}\right) \rightarrow 1$, where $\kappa_{m i c(2)}$ solves $F_{M I C(2)}(\kappa)=0$. If $C_{T} \rightarrow \infty$ and $C_{T}^{3} / T \rightarrow$ $\infty, k_{m i c} /\left(2 Z_{1} T\right)^{1 / 3} \rightarrow 1$.

Provided $C_{T}$ is constant (as is the case with Akaike's value of 2), both the $I C$ and the $M I C$ will choose $k$ proportional to $T^{1 / 2}$ asymptotically, much faster than the logarithmic rate attained under the standard asymptotic framework. The rate of $\sqrt{T}$ is optimal for unit root tests because this is the rate that will render the class of $D F$ as well as the $M$ tests non-divergent under the null hypothesis in this local asymptotic framework. However, when $C_{T}$ increases with $T$, this optimal rate is not achieved.

The proportionality factor for the rate of increase of $k_{i c}$ when $C_{T}$ increases with $T$ is one and highlights a major shortcoming of the $I C$ in such cases, namely a complete independence to $\delta$, the parameter of the local asymptotic framework. For the $k_{m i c}$ when $C_{T}$ increases with $T$, the proportionality factor depends on the parameters of the model via $Z_{1}$. This effect, however, still vanishes as $T$ increases. This lack of flexibility does not arise when $C_{T}$ is fixed since $\kappa_{m i c}$ and $\kappa_{i c}$ always maintain a dependence on $\delta$. Although $k_{i c}$ increases at the appropriate rate of $\sqrt{T}$, it too has drawbacks because $\kappa_{i c}$ is invariant to $T$ and it is the same whether $p=0$ or $p=1$. In contrast, $\kappa_{\text {mic }}$ adapts to the sample size and the trend function via a second order factor involving $Z_{1}$. Thus, the $M I C$ with $C_{T}$ fixed maintains a proportionality factor that is the most flexible with respect to $T$, the regression model, as well as parameters of the DGP. 
If the weight $C_{T}$ on the penalty factor is too large, that is $C_{T}^{3} / T \rightarrow \infty$, the $M I C$ chooses a rate that is independent of $C_{T}$, namely $T^{1 / 3}$, while the $I C$ continues to select a $k$ that is proportional to $\sqrt{T / C_{T}}$. This, however, is a result of theoretical rather than practical interest since almost all information criteria that have been suggested are such that $C_{T}^{3} / T \rightarrow 0$.

To illustrate these properties, we consider the $M A I C$ and $A I C$ with $C_{T}=2$ and the $M B I C$ and $B I C$ with $C_{T}=\ln (T)$. We denote the proportionality factors by $\kappa_{\text {maic }}, \kappa_{a i c}, \kappa_{m b i c}$ and $\kappa_{b i c}$, respectively. Numerical values of $\kappa$ are reported in Table 3.a for selected values of $\delta$ and $T$ under the null hypothesis of a unit root with $c=0$. In the case of the $M I C$ these values are random variables because of the presence of $Z_{1}$. The numerical calculations reported use its expected value. ${ }^{6}$ Table 3.a shows first that the $\kappa_{m i c} \mathrm{~S}$ are drastically larger than the $\kappa_{i c} \mathrm{~S}$ when $\delta$ is small. Second, the $\kappa_{i c} \mathrm{~S}$ vary much less with $\delta$ than the $\kappa_{m i c}$ s. Third, the $\kappa_{m i c}$ s are larger when $p=1$ than $p=0$, but the $\kappa_{i c} \mathrm{~s}$ are invariant to the order of the deterministic terms in the regression.

Values of $k$ selected by the $I C$ and the $M I C$ are in Table 3.b. These are constructed using the expected value of the $M I C$ given by (for the $I C(k)$ the term with $Z_{1}$ is absent)

$$
E[M I C(k)]=\ln \left(\sigma_{e}^{2}\right)+\frac{\left(1-\theta_{T}^{2}\right)\left(-\theta_{T}\right)^{2(k+1)}}{1-\left(-\theta_{T}\right)^{2(k+1)}}+C_{T}\left(\left[\frac{\left(1-\theta_{T}^{2}\right)\left(-\theta_{T}\right)^{2 k}}{1-\left(-\theta_{T}\right)^{2(k+1)}}\right]^{2} E\left(Z_{1}\right)+\frac{k}{T}\right)
$$

This expression is valid uniformly in $0<k \leq K_{3 T}$ for $K_{3 T}=o(T)$ and is obtained using equation (A.4) and Lemma A.5. When $\delta$ is small and especially when $p=1$, the $k_{m i c}$ are several folds larger than the $k_{i c}$. As well, differences between $k_{a i c}$ and $k_{m a i c}$ are non-trivial even for sample sizes deemed unrealistically large. Table 3.b also provides some guidance for the $k$ s that would likely be selected in practice. When $\theta=-.9$, for example, the local asymptotic results for $\delta=1$ and $T=100$ suggest that $k_{b i c}=4$ for both $p=0$ and 1 , but that the $k_{\text {maic }}$ are 8 and 13 , respectively. The local asymptotic results therefore support the earlier claim that the $I C$ lacks the flexibility to handle DGPs with a large negative moving average root and/or alternative deterministic components. The issue of practical interest is whether in finite samples and for realistic values of the moving average parameter, such large discrepancies remain and what are the implications for unit root tests. The rest of the analysis presents evidence to this effect.

\subsection{The Finite Sample Properties of $k_{m i c}$}

To evaluate the finite samples properties of the $M I C$ vis-a-vis the $I C$, we performed the following simulation experiment. For a given DGP, we construct the $\bar{M} Z_{\alpha}^{G L S}$ and $D F^{G L S}$ tests at each $k \in[0,10]$, and record the exact sizes. We then find the so-called optimal values of $k^{*}$, denoted $k_{m z}^{*}$

\footnotetext{
${ }^{6}$ To get the expected value we use the following crude approximation. For $p=0, E\left[Z_{1}\right] \approx(E[1+$ $\left.\left.\delta^{2} \int_{0}^{1} W(r)^{2} d r\right]\right) / \delta^{4} E\left(\left[\int_{0}^{1} W(r)^{2} d r\right]^{2}\right)=\left(4+2 \delta^{2}\right) / \delta^{4}$. For $p=1, E\left[Z_{1}\right] \approx E\left[1+\delta^{2} \int_{0}^{1} V_{0, \bar{c}}(r)^{2} d r\right] / \delta^{4} E\left(\int_{0}^{1} V_{0, \bar{c}}(r)^{2} d r\right)^{2}$. For $\bar{c}=-13.5$, tedious but straightforward algebra gives $E\left[Z_{1}\right] \approx\left(1+\delta^{2}(.10275) /\left(1+\delta^{4}(.01275)^{2}\right)\right.$.
} 
and $k_{d f g l s}^{*}$, as the first $k$ with a size closest to within .03 of the nominal size of 0.05 . If no such $k$ exists, $k^{*}$ is the $k$ for which the exact size of the test has the smallest absolute deviation from the nominal size of $5 \%$. We then obtain $k_{b i c}$ as the median value selected by the $B I C$ over the range 0 and 10. A similar procedure is used to obtain $k_{m b i c}, k_{a i c}$, and $k_{m a i c}$. The setup for the simulations is as outlined in Section 4.

In Table 4.a $(p=0)$ and 4.b $(p=1)$, we report the values for $k_{m z}^{*}, k_{d f g l s}^{*}, k_{i c}$ and $k_{m i c}$ for selected parameters for $C_{T}=2$ and $C_{T}=\ln \left(T-k_{\max }\right)$. While the $k_{i c}$ 's are in the same range as the $k^{*}$ 's for many cases, there are important differences between the two when $\theta<0$. The $k_{\text {mic }}$ is much closer to $k_{m z}^{*}$ and $k_{d f g l s}^{*}$ in negative $M A$ models than the $k_{i c}$. For example, when $\theta=-.8$, $k_{m z}^{*}$ and $k_{d f g l s}^{*}$ are 10 at $T=250$ and $p=1$; the $B I C$ chooses 3 over half the time whereas $k_{\text {maic }}$ has 8 as the median value. The $k_{a i c}$ is closer to $k_{m z}^{*}$ and $k_{d f g l s}^{*}$ than the $k_{b i c}$, but still not as close as the $k_{\text {maic }}$. For the $A R$ case, the $k_{\text {maic }}$ indeed selects the appropriate order of one, but when $p=1$, this sometimes differs from $k_{m z}^{*}$. This does not reflect a weakness of the $M I C$ but rather that the $\bar{M}^{G L S}$ tests are undersized when the autoregressive coefficient is large and negative. The results show that $k_{\text {maic }}$ corresponds closely to the $k_{d f g l s}^{*}$ in the AR case.

\section{Size and Power of the Tests in Finite Samples}

In this section, we need to establish two things. First, that the $M I C$ chooses values of $k$ that are appropriate in the sense of minimizing size distortions under the null hypothesis and does not over-parameterize under the alternative. Second, that the $M^{G L S}$ and $\bar{M}^{G L S}$ statistics have power comparable to the $D F^{G L S}$ and dominate the $M$ tests. Whenever an estimate of $\sigma^{2}$ is required, $s_{A R}^{2}$ based upon the regression (8) is used. Because the MAIC dominates all other criteria from both theoretical and numerical perspectives we only consider the $M^{G L S}$ tests constructed using the $M A I C$. In the simulations, the lower bound is always zero to reduce the chance of overparameterizing when a large $k$ is not necessary. The upper bound is $k_{\max }=\operatorname{int}\left(12(T / 100)^{1 / 4}\right){ }^{7}$ The results are tabulated for $T=100,150$, and 250. The ten statistics being considered are detailed in the notes to Table 5.a and 6.a.

For $p=0$, the results for size are reported in Table 5.a. The $M$ and $M^{G L S}$ tests still have inflated sizes at $T=100$ when $\theta=-0.8$. However, use of $G L S$ detrended data to construct $s_{A R}^{2}$ produces substantial size improvements. The $D F^{G L S}$ based on the $B I C$ yields an exact size around 0.4 at $\theta=-0.8$, but the $M A I C$ improves the size to 0.107 . The $M A I C$ also yields a substantial size improvement over $D F^{G L S}(t)$, which has a size of .35 when $\theta=-0.8$. The results also confirm that the $P_{T}$ test constructed with $k$ chosen by $B I C$ suffers from substantial size distortions. However,

\footnotetext{
${ }^{7}$ In practice, the upper bound should be set such that the constraint does not bind. In simulations, the upper bound occasionally binds when $T=100$ but not for larger sample sizes (e.g. $T=150$ ).
} 
the $\bar{P}_{T}$ and $\bar{M} P_{T}^{G L S}$ show remarkably little size distortions when the $M A I C$ is used to select $k$.

To assess the relative power of the tests, we tabulate the rejection rates at $\bar{\alpha}=1+\bar{c} / T$. For $p=0$ and $\bar{c}=-7.0$, the values of $\bar{\alpha}$ are $0.93,0.953$ and 0.972 for $T=100,150$ and 250, respectively. ${ }^{8}$ The size-adjusted power of the tests are reported in Table 5.b. Although the tests should reject the null hypothesis $50 \%$ of the time in large samples, none of the tests quite achieve the $50 \%$ (asymptotic) target for sample sizes less than 200. Thus, in finite samples, the need to account for serial correlation reduces the power of the tests across the board. However, the GLS based tests have more power than tests which do not implement GLS detrending. Furthermore, the power increase from $T=100$ to $T=150$ is quite substantial, especially when we take into account of the fact that we are evaluating at a closer alternative at $T=150$ than at $T=100$.

For $p=1$, the results for size are reported in Table 6.a. The size distortions are somewhat larger when there is a linear trend in the regression, but there are clearly gains from using $G L S$ detrended data to construct $s_{A R}^{2}$ and selecting $k$ with the $M A I C$. The resulting $\bar{M}^{G L S}$ tests have size close to the nominal size for all parameter values, while the $M$ and $M^{G L S}$ tests continue to have size close to .15. As well, the $M A I C$ yields exact sizes for the $D F^{G L S}$ and the $P_{T}$ tests that are far more accurate than those obtained using the sequential $t$ test and the $B I C$.

The size-adjusted power of the tests for $p=1$ are reported in Table 6.b. Power is again evaluated at $\bar{\alpha}=1+\bar{c} / T$. For $\bar{c}=-13.5, \bar{\alpha}$ is 0.865 for $T=100,0.910$ for $T=150$, and 0.946 for $T=250$. Compared to the size-adjusted power reported in Table 5.b, all GLS based tests have lower power when $p=1$. However, there are non-negligible power gains from $G L S$ detrending.

A feature of interest is that the $M I C$ allows the $\bar{M}^{G L S}$ tests to have an exact size that is closer to $5 \%$ than fixing $k$ at $k_{m z}^{*}$ assuming a known DGP. For example, in the $M A$ case with $\theta=-.8$ and $p=1$, the size is .059 using the $M I C$ and .159 using a value of $k$ fixed at $k_{m z}^{*}=6$. When $T=150$, the corresponding values are .027 with the $M A I C$ and .093 at $k_{m z}^{*}=8$ (not reported). Hence, the $M A I C$ chooses a $k$ that adapts to the particular sample analyzed. Size improvements obtained using the $M I C$ compared to fixing $k$ at $k^{*}$ are even larger when the number of deterministic components increases (see Perron and Rodríguez (1998) for the case of a trend with a change in slope).

The size reported for $\bar{M}^{G L S}$ is remarkable, given that when $\theta<0$, the $Z$ tests based on kernel estimates of $\sigma^{2}$ reject with probability close to one under the null hypothesis. The $D F$ tests have higher size-unadjusted power in the case of negative $M A$ errors. Such high probabilities of rejections are misleading, however, as they reflect size distortions. Although the $D F_{b i c}^{G L S}$ has higher size-adjusted power than $\bar{M}_{m i c}^{G L S}$ for some parameter values, this result must also be put into

\footnotetext{
${ }^{8}$ We also performed extensive power simulations with other values of $\alpha$ and $T$. The results are qualitatively similar and do not affect the conclusions concerning the relative performance of the different tests.
} 
perspective. Consider $\theta=-0.8, T=100$ and $p=1$. While the size-adjusted power of the $D F_{b i c}^{G L S}$ is higher than the $\bar{M}_{m i c}^{G L S}$ by 5 basis points, its size is more distorted by 45 basis points. This is a substantial increase in the probability of a Type I error. The $D F_{m i c}^{G L S}$ fares better, but there is still a size advantage in favor of the $\bar{M}_{m i c}^{G L S}$.

Overall, the $\bar{M}_{m i c}^{G L S}$ tests have better sizes, while the $D F_{m i c}^{G L S}$ has better power. A drawback of the $\bar{M}_{m i c}^{G L S}$ test is that it is substantially undersized for pure $A R$ processes with a coefficient close to -1 . Although economic time series rarely display such a property, this nonetheless translates into lower (size unadjusted) power. As discussed in Section 6.2, this is not due to a weakness of the $M I C$ in selecting $k$, but rather is an inherent property of the $\bar{M}^{G L S}$ tests. The trade-offs between the $D F_{m i c}^{G L S}$ and the $\bar{M}_{\text {mic }}^{G L S}$ are to be determined by the practitioner.

\section{Empirical Illustrations.}

To illustrate the ease of application of our procedures and the differences in inference that can result, we consider inflation series from the G7 countries. Data for the GDP deflator are taken from the OECD International Sector Database. We constructed annualized quarterly inflation rate series covering the period 1960:2 to 1997:2 ( $T+1=149$ observations) as (400 times) the logged differences of successive quarters. All estimation results are presented in Table 7. As a preliminary step, we first estimated simple $A R M A(1,1)$ model for each series. The results show autoregressive roots that are indeed close to one (ranging from .92 for Germany to .98 for France). Also, the MA coefficients are negative for all series, ranging from -.27 for Italy to -.72 for France. This suggests that such series are likely affected by the types of problems we discussed.

The first step to constructing the tests is to generate $G L S$ detrended series as defined by (6) with $\bar{c}=-7$ as the non-centrality parameter (since inflation series are non-trending; otherwise use $\bar{c}=-13.5)$. The next step is to estimate by $O L S$ the autoregression (8) for all values of $k$ ranging from 0 to some maximal order, say $k_{\max }$. We use $k_{\max }=\operatorname{int}\left(12(T / 100)^{1 / 4}\right)$ but other values are valid. In the model selection stage, each autoregression uses the same number of effective observations, namely $T-k_{\max }$. The selected $k_{\text {maic }}$ is obtained as the value of $k$ which minimizes (12). Given $k_{\text {maic }}$, the $D F_{\text {maic }}^{G L S}$ is obtained as the t-statistic on $\beta_{0}=0$ from re-estimation of (8) with $T-k_{\text {maic }}$ effective observations. The same regression is used to construct $s_{A R}^{2}$ according to (5). Then, the $M^{G L S}$ tests are constructed from (3) and (4), the $P_{T}^{G L S}$ test from (7), and the $M P_{T}^{G L S}$ test from (9), all with $G L S$ detrended series $\widetilde{y}_{t}$. Using the $B I C$, the steps are the same except that the term $\tau_{T}(k)$ is omitted from (12).

The results show interesting differences between using the $M A I C$ or the $B I C$ to select the lag length. First, for all countries, except the U.S., the selected lag length is higher with $M A I C$ than with BIC. Second, in all cases (except the U.S.), the evidence in favor of stationarity is weaker 
with the $M A I C$ than with the $B I C$. For example, with the $B I C$, the $M Z_{\alpha, b i c}^{G L S}$ and $D F_{b i c}^{G L S}$ tests suggest a rejection at the $1 \%$ significance level for Japan and Germany, at the $5 \%$ level for Canada, and at the $10 \%$ level for Italy. When using the $M A I C$, there are only rejections at the $5 \%$ level for Germany (showing that the tests still have power) and at the $10 \%$ level for the U.K. For Canada, the $D F_{\text {maic }}^{G L S}$ is significant at the $10 \%$ level but barely. For the U.S. and France, there is no difference in outcome. Of interest also is the fact that the $\bar{Z}_{\alpha, \text { maic }}^{G L S}$ still shows strong rejections at the $1 \%$ level for Japan, the U.K. and Germany. This accords with the simulation results that the $\bar{Z}_{\alpha}^{G L S}$ test has high size distortions even when constructed using the $M A I C$.

\section{Conclusion}

The analysis of ERS shows that detrending data by $G L S$ yields power gains for unit root tests. We find that $G L S$ detrending also allows for a more precise autoregressive spectral density estimate and ensures that it is invariant to the parameters of the trend function. We also show that the $B I C$ or $A I C$ are not sufficiently flexible for unit root tests. We suggest a modification, the $M A I C$, which is evidently more robust when there are negative moving-average errors. Use of the $M A I C$ in conjunction with $G L S$ detrended data results in a class of $\bar{M}^{G L S}$ tests that have good size and power. The key distinction between the $M A I C$ and standard information criteria is that the former takes account of the fact that the bias in the estimate of the sum of the autoregressive coefficients is highly dependent on $k$. In this paper, we show that the $M A I C$ is useful for the $D F^{G L S}$ test and the construction of the autoregressive estimate of the spectral density at frequency zero. We also expect the $M A I C$, or the more general $M I C$, to be useful in a broader range of applications because macroeconomic data are known to be highly persistent, and in such cases, the bias in the estimate of the sum of the autoregressive coefficients should depend on the order of the autoregression. However, it should also be noted that while the suggested information criterion is useful in the context of unit root tests as we have shown, it may not be appropriate when selecting the lag order in other contexts. The general usefulness of the $M I C$ also awaits further investigation. 


\section{References}

Berk, K. N. (1974), Consistent Autoregressive Spectral Estimates, The Annals of Statistics 2, 489502.

DeJong, D. N. , J. C. Nankervis, N. E. Savin and C. H. Whiteman (1992), The Power Problem of Unit Root Tests in Time Series with Autoregressive Errors, Journal of Econometrics 53, 323343.

Dickey, D. A. and Fuller, W. A. (1979), Distribution of the Estimators for Autoregressive Time Series with a Unit Root, Journal of the American Statistical Association 74, 427-431.

Dufour, J. M. and King, M. (1991), Optimal Invariant Tests for the Autocorrelation Coeficient in Linear Regressions with Stationary and Nonstationary Errors, Journal of Econometrics 47, 115-143.

Elliott, G., Rothenberg, T. J. and Stock, J. H. (1996), Efficient Tests for an Autoregressive Unit Root, Econometrica 64, 813-836.

Franses, P. H. and Haldrup, N. (1994), The Effects of Additive Outliers on Tests of Unit Roots and Cointegration, Journal of Business and Economic Statistics 12, 471-478.

Fuller, W. A. (1976), Introduction to Statistical Time Series, John Wiley, New York.

Gourieroux, C. and Monfort, A. (1995), Statistical and Econometric Models, Vol. 1, Cambridge Unversity Press, Cambridge.

Hannan, E. J. and Deistler, M. (1988), The Statistical Theory of Linear Systems, John Wiley, New York.

Lopez, J. H. (1997), The Power of the ADF Test, Economics Letters 57, 5-10.

Nabeya, S. and Perron, P. (1994), Local Asymptotic Distribution Related to the AR(1) Model with Dependent Errors, Journal of Econometrics 62, 229-264.

Ng, S. and Perron, P. (1995), Unit Root Tests in ARMA Models with Data Dependent Methods for the Selection of the Truncation Lag, Journal of the American Statistical Association 90, 268281.

Ng, S. and Perron, P. (2000), A Note on the Selection of Time Series Models, manuscript, Department of Economics, Boston College. 
Pantula, S. G. (1991), Asymptotic Distributions of Unit Root Tests When the Process is Nearly Stationary, Journal of Business and Economic Statistics 9, 63-71.

Perron, P. and Ng, S. (1998), An Autoregressive Spectral Density Estimator at Frequency Zero for Nonstationarity Tests, Econometric Theory 14, 560-603.

Perron, P. and Ng, S. (1996), Useful Modifications to Unit Root Tests with Dependent Errors and their Local Asymptotic Properties, Review of Economic Studies 63, 435-465.

Perron, P. and Rodríguez, G. (1998), GLS Detrending, Efficient Unit Root Tests and Structural Change, manuscript, Boston University.

Phillips, P. C. B. (1987), Time Series Regression with Unit Roots, Econometrica 55, 277-302.

Phillips, P. C. B. and Perron, P. (1988), Testing for a Unit Root in Time Series Regression, Biometrika 75, 335-346.

Press, W. H., Teukolsky, S., Vetterling, W. and Flannery, B. (1992), Numerical Recipies in C, second edn, Cambridge University Press, Cambridge.

Said, S. E. and Dickey, D. A. (1984), Testing for Unit Roots in Autoregressive-Moving Average Models of Unknown Order, Biometrika 71, 599-607.

Schwert, G. W. (1989), Tests for Unit Roots: A Monte Carlo Investigation, Journal of Business and Economic Statistics 7, 147-160.

Stock, J. H. (1990), A Class of Tests for Integration and Cointegration, manuscript, Harvard University. 


\section{Appendix}

\section{Proof of Theorem 2}

In this Appendix, for simplicity, we consider the derivations in the case where the DGP is (14) and no deterministic component is present in the regression (8), in which case $y_{t}$ is used instead of $\widetilde{y}_{t}$. All the results carry over to the more general case with least-squares or GLS detrending. With $G L S$ detrending, the stated results remain exactly the same when $p=0$ and are as stated in the text with $V_{c, \bar{c}}(r)$ replacing $J_{c}(r)$ when $p=1$. As a matter of notation, we let $\Rightarrow$ denote weak convergence in distribution and $\rightarrow$ convergence in probability. Also, to alleviate notational burden, we let $N=T-k_{\max }$. Note that we can write the DGP as

$$
\Delta y_{t}=-\sum_{i=1}^{k}\left(-\theta_{T}\right)^{i} \Delta y_{t-i}+\frac{c}{T} \sum_{i=0}^{k}\left(-\theta_{T}\right)^{i} y_{t-i-1}+e_{t}-\left(-\theta_{T}\right)^{k+1} e_{t-k-1} .
$$

Let

$$
z_{t-j}=\sum_{i=0}^{k-j}\left(-\theta_{T}\right)^{i} \Delta y_{t-i-j}=\frac{c}{T} \sum_{i=0}^{k-j}\left(-\theta_{T}\right)^{i} y_{t-i-j-1}+e_{t-j}-\left(-\theta_{T}\right)^{k+1-j} e_{t-k-1},
$$

the DGP can also be written as

$$
\begin{aligned}
\Delta y_{t} & =\frac{c}{T} y_{t-1}-\left(-\theta_{T}\right) z_{t-1}+\frac{c}{T} \sum_{i=1}^{k}\left(-\theta_{T}\right)^{i} y_{t-i-1}+e_{t}-\left(-\theta_{T}\right)^{k+1} e_{t-k-1} \\
& =\beta_{0} y_{t-1}+\sum_{i=1}^{k} \beta_{i} z_{t-i}+e_{t k}
\end{aligned}
$$

with $\beta_{0}=c / T, \beta_{1}=\theta_{T}, \beta_{i}=0$ for $i>1$, and

$$
e_{t k}=\frac{c}{T} \sum_{i=1}^{k}\left(-\theta_{T}\right)^{i} y_{t-i-1}+e_{t}-\left(-\theta_{T}\right)^{k+1} e_{t-k-1}
$$

Since $\left\{z_{t-i}, i=1, \ldots, k\right\}$ is a linear function of $\left\{\Delta y_{t-i}, i=1, \ldots, k\right\}$, the $O L S$ estimate of $\beta_{0}$ and the sum of squared residuals from the regression

$$
\Delta y_{t}=\beta_{0} y_{t-1}+\sum_{i=1}^{k} \beta_{i} \Delta y_{t-i}+e_{t k}
$$

have the same properties as the corresponding quantities from estimating (A.1). We start with a Lemma that will be used extensively.

Lemma A.1 Let $J_{c}(r)=\int_{0}^{r} \exp ((r-s) c) d W(s), K_{3 T}=o(T)$ and $\delta_{i j}=1$ if $i=j$ and 0 otherwise with $i, j=1, \ldots, k$. Then, uniformly for $i, j \leq k_{\max } \leq K_{3 T}=o(T)$ : a) $N^{-1} \sum_{t=k_{\max }+1}^{T} y_{t-1}^{2}=\sigma_{e}^{2}(1+$ $\left.\delta^{2} \int_{0}^{1} J_{c}(r)^{2} d r\right)+O_{p}\left(T^{-1 / 2}\right)$; b) $\left.N^{-1} \sum_{t=k_{\max }+1}^{T} y_{t-1} z_{t-j}=\delta_{1 j} \sigma_{e}^{2}+O_{p}\left(T^{-1 / 2}\right) ; c\right) N^{-1} \sum_{t=k_{\max }+1}^{T} z_{t-i} z_{t-j}=$ $\left.\sigma_{e}^{2}\left(\delta_{i j}+\left(-\theta_{T}\right)^{2(k+1)-i-j}\right)+O_{p}\left(T^{-1 / 2}\right) ; d\right) N^{-1} \sum_{t=k_{\max }+1}^{T} \Delta y_{t} y_{t-1}=-\sigma_{e}^{2}+O_{p}\left(T^{-1 / 2}\right)$;

e) $N^{-1} \sum_{t=k_{\max }+1}^{T} \Delta y_{t} z_{t-j}=-\delta_{1 j} \sigma_{e}^{2}+O_{p}\left(T^{-1 / 2}\right)$. 
Proof: From Nabeya and Perron (1994), we can write $y_{t}=a_{T} e_{t}+b_{T} X_{t}$ where $X_{t}=(1+$ $c / T) X_{t-1}+e_{t}, a_{T}=\left(-\theta_{T}\right)(1+c / T), b_{T}=1-(1+c / T)\left(-\theta_{T}\right), a_{T} \rightarrow 1, T^{1 / 2} b_{T} \rightarrow \delta$ as $T \rightarrow \infty$. Also, $\sum_{t=1}^{T} X_{t-1} e_{t}=O_{p}(T)$. For part(a), we have:

$$
\begin{aligned}
& N^{-1} \sum_{t=k_{\max }+1}^{T} y_{t-1}^{2} \\
= & N^{-1} \sum_{t=k_{\max }+1}^{T}\left(a_{T}^{2} e_{t-1}^{2}+b_{T}^{2} X_{t-1}^{2}+2 a_{T} b_{T} X_{t-1} e_{t-1}\right)=\sigma_{e}^{2}\left(1+\delta^{2} \int_{0}^{1} J_{c}(r)^{2} d r\right)+O_{p}\left(T^{-1 / 2}\right) .
\end{aligned}
$$

For part (b), we have

$$
\begin{aligned}
& N^{-1} \sum_{t=k_{\max }+1}^{T} y_{t-1} z_{t-j} \\
= & N^{-1} \sum_{t=k_{\max }+1}^{T}\left(a_{T} e_{t-1}+b_{T} X_{t-1}\right)\left(\frac{c}{T} \sum_{i=0}^{k-j}\left(-\theta_{T}\right)^{i} y_{t-i-j-1}-\left(-\theta_{T}\right)^{k+1-j} e_{t-k-1}+e_{t-j}\right) .
\end{aligned}
$$

Since $X_{t}=\sum_{j=1}^{t} \exp ((t-j) c / T) e_{j}, X_{t-1}=X_{t-k-1}+\sum_{j=1}^{k} \exp ((t-j) c / T) e_{j}$. We have $\sum_{t=k_{\max }+1}^{T} e_{t} e_{s}=$ $O_{p}\left(T^{1 / 2}\right)$ for $t \neq s, T^{1 / 2} b_{T}=O_{p}(1)$, and $\sum_{t=k_{\max }+1}^{T} X_{t-1} e_{t}=O_{p}(T)$, hence, $N^{-1} \sum_{t=k_{\max }+1}^{T} y_{t-1} z_{t-1}-$ $\sigma_{e}^{2}=O_{p}\left(T^{-1 / 2}\right)$ and $N^{-1} \sum_{t=k_{\max }+1}^{T} y_{t-1} z_{t-j}=O_{p}\left(T^{-1 / 2}\right)$ for $j>1$ uniformly in $0<i, j \leq k_{\max } \leq$ $K_{3 T}$. For part (c),

$$
\begin{aligned}
& N^{-1} \sum_{t=k_{\max }+1}^{T} z_{t-i} z_{t-j} \\
= & N^{-1} \sum_{t=k_{\max }+1}^{T}\left[e_{t-i}-e_{t-k-1}\left(-\theta_{T}\right)^{k+1-i}\right]\left[e_{t-j}-e_{t-k-1}\left(-\theta_{T}\right)^{k+1-j}\right]+O_{p}\left(T^{-1 / 2}\right) \\
= & \sigma_{e}^{2}\left(\delta_{i j}+\left(-\theta_{T}\right)^{2(k+1)-i-j}\right)+O_{p}\left(T^{-1 / 2}\right) .
\end{aligned}
$$

Part (d) follows using the fact that

$$
N^{-1} \sum_{t=k_{\max }+1}^{T} \Delta y_{t} y_{t-1}=c N^{-2} \sum_{t=k_{\max }+1}^{T} y_{t-1}^{2}+N^{-1} \sum_{t=k_{\max }+1}^{T}\left(a_{T} e_{t-1}+b_{T} X_{t-1}\right)\left(e_{t}+\theta_{T} e_{t-1}\right),
$$

and part (e) using the fact that

$$
\begin{aligned}
& N^{-1} \sum_{t=k_{\max }+1}^{T} \Delta y_{t} z_{t-i} \\
= & N^{-1} \sum_{t=k_{\max }+1}^{T}\left(\frac{c}{T} y_{t-1}+e_{t}-\left(-\theta_{T}\right) e_{t-1}\right)\left(\frac{c}{T} \sum_{j=0}^{k-i}\left(-\theta_{T}\right)^{j} y_{t-i-j-1}+e_{t-i}-e_{t-k-1}\left(-\theta_{T}\right)^{k+1-i}\right) .
\end{aligned}
$$

The proof of Theorem 2 follows directly from the following Lemma concerned with the limit of $\hat{\beta}_{0}$, the $O L S$ estimate of $\beta_{0}$ from the regression (A.1) which is equivalent to the $O L S$ estimate of $\beta_{0}$ from the autoregression (8). 
Lemma A.2 Consider the OLS estimate of $\beta_{0}$ from the autoregression (8). Let $x=\delta^{2} \int_{0}^{1} J_{c}(r)^{2} d r$ when $p=0$ and $x=\delta^{2} \int_{0}^{1} V_{c, \bar{c}}(r)^{2} d r$ when $p=1$. Then, 1) uniformly in $0<k \leq K_{1 T}: k \hat{\beta}_{0} \Rightarrow$ $-x^{-1}$; 2) if $k / T^{1 / 2} \rightarrow \kappa, T^{1 / 2} \hat{\beta}_{0} \Rightarrow A$, where $A$ is a random variable with $E(A)=-\vartheta(\kappa) E\left(x^{-1}\right)$, $\vartheta(\kappa)=2 \delta \exp (-2 \kappa \delta) /(1-\exp (-2 \kappa \delta))$; 3) uniformly in $K_{2 T} \leq k \leq K_{3 T}, T^{1 / 2} \hat{\beta}_{0}=O_{p}(1)$.

Case $0<k \leq K_{1 T}$.

Let $X_{t}^{\prime}=\left(y_{t-1}, Z_{t}^{\prime}\right), Z_{t}=\left(z_{t-1}, \ldots, z_{t-k}\right)^{\prime}$. Define the following two $(k+1)$ by $(k+1)$ matrices:

$$
\begin{gathered}
\hat{R}_{T}=N^{-1} \sum_{t=k_{\max }+1}^{T} X_{t} X_{t}^{\prime}=N^{-1}\left[\begin{array}{cc}
\sum_{t=k_{\max }+1}^{T} y_{t-1}^{2} & \sum_{t=k_{\max }+1}^{T} y_{t-1} Z_{t}^{\prime} \\
\sum_{t=k_{\max }+1}^{T} Z_{t} y_{t-1} & \sum_{t=k_{\max }+1}^{T} Z_{t} Z_{t}^{\prime}
\end{array}\right] \\
\widetilde{R}=\sigma_{e}^{2}\left[\begin{array}{ll}
1+x & e^{\prime} \\
e & I_{k}+\iota \iota^{\prime}
\end{array}\right]
\end{gathered}
$$

where $x=\delta^{2} \int_{0}^{1} J_{c}(r)^{2} d r, e^{\prime}=\left(1,0_{1 \times k}\right)$ and $\iota$ is a $k \times 1$ vector of ones. Using Maple, we obtained the inverse:

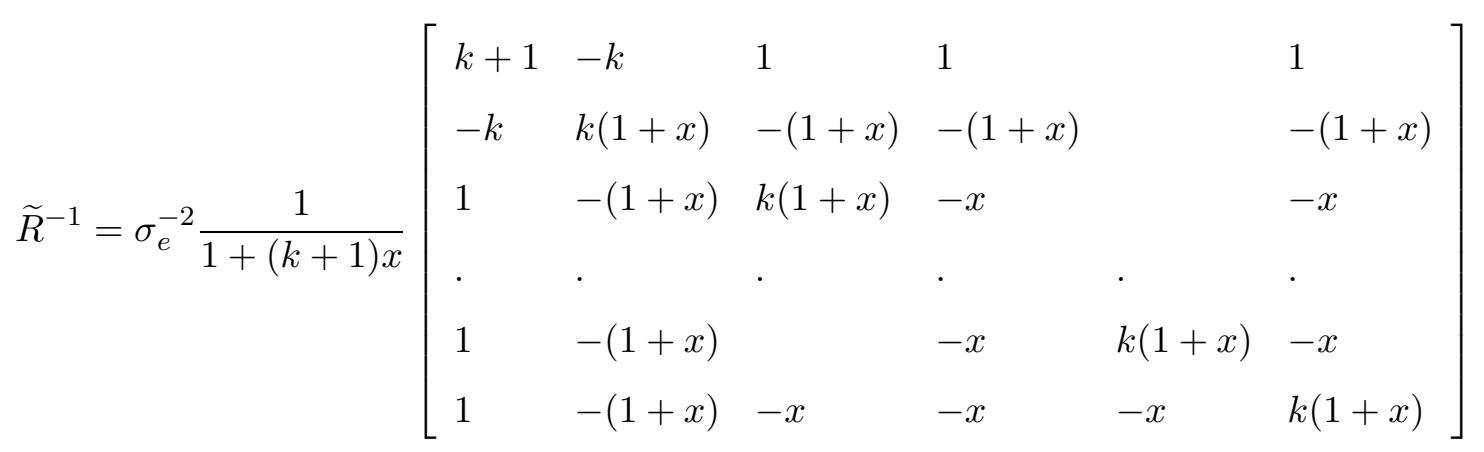

In what follows, the structure of $\widetilde{R}^{-1}$ will be exploited. Let

$$
\hat{V}_{T}=\left(N^{-1} \sum_{t=k_{\max }+1}^{T} \Delta y_{t} y_{t-1}, N^{-1} \sum_{t=k_{\max }+1}^{T} \Delta y_{t} Z_{t}^{\prime}\right)^{\prime}, \quad \widetilde{V}=\left(-1,-1,0_{k-1}^{\prime}\right)^{\prime} .
$$

Note that $\hat{\theta}=\left(\hat{\beta}_{0}, \hat{\beta}_{1}, \ldots, \hat{\beta}_{k}\right)^{\prime}=\hat{R}_{T}^{-1} \hat{V}_{T}$.

Lemma A.3 a) $\max _{0<k \leq K_{1 T}}\left\|\hat{R}_{T}-\widetilde{R}\right\|=O_{p}\left(K_{1 T} / \sqrt{T}\right)$, b) $\max _{0<k \leq K_{1 T}}\left\|\hat{R}_{T}^{-1}-\widetilde{R}^{-1}\right\|=O_{p}\left(K_{1 T} / \sqrt{T}\right)$.

Proof: Using Lemma A.1, we have $\hat{R}_{i j}-\widetilde{R}_{i j}=C_{i j}$ where $C_{i, j}=O_{p}\left(T^{-1 / 2}\right)$ uniformly in $0<k \leq$ $K_{1 T}$ for $i, j=1, \ldots, k+1$ and part a) follows. To prove part b), note that the lower $k \times k$ submatrix of $\widetilde{R}$ is, say, $\widetilde{R}_{k}=\left(I_{k}+\iota \iota^{\prime}\right)$. Then $\left(I_{k}+\iota \iota^{\prime}\right)^{-1}=I_{k}-\left(1+\iota^{\prime} \iota\right)^{-1} \iota \iota^{\prime}$ has eigenvalues of 1 and $\left(1+\iota^{\prime} \iota\right)^{-1}$. 
Hence $\max _{0<k \leq K_{1 T}}\left\|\widetilde{R}_{k}^{-1}\right\|=O_{p}(1)$. Using arguments as in Perron and $\mathrm{Ng}(1998)$, the $(k+1) \times(k+$ 1) matrix $\widetilde{R}$ also has eigenvalues of the same order as $\widetilde{R}_{k}$. Hence $\max _{0<k \leq K_{1 T}}\left\|\widetilde{R}^{-1}\right\|=O_{p}(1)$. Let $Q_{T}=\left\|\hat{R}_{T}^{-1}-\widetilde{R}^{-1}\right\|$. Then $\max _{0<k \leq K_{1 T}} Q_{T} \leq \max _{0<k \leq K_{1 T}}\left\|\hat{R}_{T}^{-1}\left|\left\|\mid \hat{R}_{T}-\widetilde{R}\right\|\left\|\widetilde{R}^{-1}\right\|=O_{p}\left(K_{1 T} / \sqrt{T}\right)\right.\right.$ by arguments analogous to Berk (1974).

Lemma A.4 a) $\left.\max _{0<k \leq K_{1 T}}|| \hat{V}_{T}-\widetilde{V} \|=O_{p}\left(\sqrt{K_{1 T} / T}\right) ; b\right) \max _{0<k \leq K_{1 T}}\left\|\hat{V}_{T}\right\|=O_{p}(1)$.

Proof: Part (a) follows directly from Lemma A.1 (d,e). Since $\widetilde{V}=\left(-1,-1,0_{1 \times k-1}\right)^{\prime}, \max _{0<k \leq K_{1 T}}$ $\|\widetilde{V}\|=O_{p}(1)$ and $\max _{0<k \leq K_{1 T}}\left\|\hat{V}_{T}\right\|=O_{p}(1)$ in view of part (a) of the Lemma.

Let $e^{\prime}=\left(1,0_{1 \times k}\right)$. We have

$$
\begin{aligned}
\hat{\beta}_{0} & =e^{\prime} \hat{R}_{T}^{-1} \hat{V}_{T} \\
& =e^{\prime}\left(\hat{R}_{T}^{-1}-\widetilde{R}^{-1}\right)\left(\hat{V}_{T}-\widetilde{V}\right)+e^{\prime}\left(\hat{R}_{T}^{-1}-\widetilde{R}^{-1}\right) \widetilde{V}+e^{\prime} \widetilde{R}^{-1}\left(\hat{V}_{T}-\widetilde{V}\right)+e^{\prime} \widetilde{R}^{-1} \widetilde{V} \\
& =A+B+C+D .
\end{aligned}
$$

For a matrix $M$, denote the $i, j$-th element of $M^{-1}$ by $m^{i j}$. We now consider the limit of each of the four terms. For $A$,

$$
\begin{aligned}
\max _{0<k \leq K_{1 T}}\left\|k e^{\prime}\left(\hat{R}_{T}^{-1}-\widetilde{R}\right)\left(\hat{V}_{T}-\widetilde{V}\right)\right\|^{2} & \leq K_{1 T}^{2} \sum_{i=1}^{k}\left\|\hat{r}^{1, i}-\widetilde{r}^{1, i}\right\|^{2}\left\|\hat{V}_{i, T}-\widetilde{V}_{i}\right\|^{2} \\
& \leq c \frac{K_{1 T}^{2}}{T} \sum_{i=1}^{k}\left\|\hat{r}^{1, i}-\widetilde{r}^{1, i}\right\|^{2} \leq c\left(\frac{K_{1 T}^{2}}{T}\right)^{2} \rightarrow 0
\end{aligned}
$$

since $\sum_{i=1}^{k}\left\|\hat{r}^{1, i}-\widetilde{r}^{1, i}\right\|^{2} \leq\left\|\hat{R}_{T}^{-1}-\widetilde{R}^{-1}\right\|^{2}=O_{p}\left(k^{2} / T\right) \leq O_{p}\left(K_{1 T}^{2} / T\right)$. For the term $\mathrm{B}$, we need $\max _{0<k \leq K_{1 T}}\left\|k e^{\prime}\left(\hat{R}_{T}^{-1}-\widetilde{R}^{-1}\right) \widetilde{V}\right\|$. Recall that $\hat{R}_{T}=\widetilde{R}+C$, where $C_{i j}=O_{p}\left(T^{-1 / 2}\right), i, j=1, \ldots, k+$ 1. Then $\hat{R}_{T}^{-1}=\widetilde{R}^{-1}+\widetilde{R}^{-1} C \widetilde{R}^{-1}+O_{p}\left(T^{-1}\right)$. We have (ignoring, without loss, the $O_{p}\left(T^{-1}\right)$ term):

$$
\begin{gathered}
\left\|e^{\prime}\left(\hat{R}_{T}^{-1}-\widetilde{R}^{-1}\right) \widetilde{V}\right\|=\left\|e^{\prime} \widetilde{R}^{-1} C \widetilde{R}^{-1} \widetilde{V}\right\|=\left\|\sum_{j=1}^{k+1}\left(\widetilde{r}^{1 j}+\widetilde{r}^{2 j}\right) \sum_{i=1}^{k+1} \widetilde{r}^{1 i} C_{i j}\right\| \\
\leq \sum_{j=1}^{k+1}\left\|\left(\widetilde{r}^{1 j}+\widetilde{r}^{2 j}\right)\right\| \sum_{i=1}^{k+1}\left\|\widetilde{r}^{1 i}\right\|\left\|C_{i j}\right\| \leq c^{*} \sum_{j=1}^{k}\left\|\left(\widetilde{r}^{1 j}+\widetilde{r}^{2 j}\right)\right\| \sum_{i=1}^{k+1}\left\|\widetilde{r}^{1 i}\right\|,
\end{gathered}
$$

where $c^{*}=\max _{i, j} C_{i j}$. But the sum of the norms of the elements of the first row of $\widetilde{R}^{-1}$ is $[2 k+(k-1)][1+(k+1) x]^{-1}=O_{p}(1)$, and the sum of the norms of the sum of the first two elements of each row of $\widetilde{R}^{-1}$ is given by $\frac{1+k x+(k-1) x}{1+(k+1) x}=O_{p}(1)$. Since $c^{*}=O_{p}\left(T^{-1 / 2}\right), \max _{0<k \leq K_{1 T}} \| k e^{\prime}\left(\hat{R}_{T}^{-1}-\right.$ $\left.\widetilde{R}^{-1}\right) \widetilde{V} \|=O_{p}\left(K_{1 T} / T^{1 / 2}\right)$. For the term C, first note that

$$
\left(\hat{V}_{T}-\tilde{V}\right)^{\prime}=N^{-1}\left[\left(\sum_{t=k_{\max }+1}^{T} \Delta y_{t} y_{t-1}+1\right),\left(\sum_{t=k_{\max }+1}^{T} \Delta y_{t} z_{t-1}+1\right), \sum_{t=k_{\max }+1}^{T} \Delta y_{t} z_{t-2}, \ldots, \sum_{t=k_{\max }+1}^{T} \Delta y_{t} z_{t-k}\right] .
$$


Thus,

$$
\begin{aligned}
& \max _{0<k \leq K_{1 T}}\left\|k e^{\prime} \widetilde{R}^{-1}\left(\hat{V}_{T}-\tilde{V}\right)\right\| \leq \\
& \frac{K_{1 T}}{1+\left(K_{1 T}+1\right) x}\left[\left\|\left(K_{1 T}+1\right)\left(N^{-1} \sum_{t=k_{\max }+1}^{T} \Delta y_{t} y_{t-1}+1\right)\right\|-\left\|K_{1 T}\left(N^{-1} \sum_{t=k_{\max }+1}^{T} \Delta y_{t} z_{t-1}+1\right)\right\|\right. \\
& \left.\quad+N^{-1} \sum_{j=2}^{k}\left\|\sum_{t=k_{\max }+1}^{T} \Delta y_{t} z_{t-j}\right\|\right] \leq\left(c K_{1 T} / N^{1 / 2}\right) \rightarrow 0 .
\end{aligned}
$$

For D,

$$
\max _{0<k \leq K_{1 T}}\left(k e^{\prime} \widetilde{R}^{-1} \widetilde{V}\right)=\frac{-K_{1 T}}{1+\left(K_{1 T}+1\right) x} \rightarrow \frac{-1}{x} \equiv \frac{-1}{\delta^{2} \int_{0}^{1} J_{c}(r)^{2} d r}
$$

Combining the results, we have $k \hat{\beta}_{0}=o_{p}(1)+o_{p}(1)+o_{p}(1)+O_{p}(1)=O_{p}(1)$. Thus, $k \hat{\beta}_{0} \Rightarrow$ $\left(\delta^{2} \int_{0}^{1} J_{c}(r)^{2} d r\right)^{-1}$ uniformly in $0<k \leq K_{1 T}$.

2 Case $k / T^{1 / 2} \rightarrow \kappa$.

For the case where $k / T^{1 / 2} \rightarrow \kappa$, we first note that the relevant matrix $\widetilde{R}$ is given by

$$
\sigma_{e}^{2}\left[\begin{array}{ll}
1+x & e^{\prime} \\
e & I_{k}+\mu \mu^{\prime}
\end{array}\right]
$$

where $\mu^{\prime}=\left[\left(-\theta_{T}\right)^{k}, \ldots,\left(-\theta_{T}\right)\right]$. The limiting vector $\tilde{V}$ remains unchanged. Now we have $\sqrt{T} \hat{\beta}_{0}=$ $\sqrt{T} e^{\prime} \widetilde{R}^{-1} \widetilde{V}+\sqrt{T} W$, where $W=A+B+C$. It is easy to see that $E(\sqrt{T} W)=0$ in large samples. So, we need to evaluate $\sqrt{T} e^{\prime} \widetilde{R}^{-1} \widetilde{V}$, (minus) the sum of the first two elements of $\widetilde{R}^{-1}$. By partitioned matrix inversion, these are given by

$$
\begin{aligned}
& \widetilde{R}_{11}^{-1}:\left[(1+x)-e^{\prime}\left(I_{k}+\mu \mu^{\prime}\right)^{-1} e\right]^{-1}, \\
& \widetilde{R}_{12}^{-1}:-\left[(1+x)-e^{\prime}\left(I_{k}+\mu \mu^{\prime}\right)^{-1} e\right]^{-1} e^{\prime}\left(I_{k}+\mu \mu^{\prime}\right)^{-1} e .
\end{aligned}
$$

Since $\left(I_{k}+\mu \mu^{\prime}\right)^{-1}=I_{k}-\mu \mu^{\prime} / \lambda, \lambda=1+\mu^{\prime} \mu, e^{\prime}\left(I_{k}+\mu \mu^{\prime}\right)^{-1} e=e^{\prime} e-\left(e^{\prime} \mu \mu^{\prime} e / \lambda\right)=1-\left[\left(-\theta_{T}\right)^{2 k} / \lambda\right]$. Hence, $1-e^{\prime}\left(I_{k}+\mu \mu^{\prime}\right)^{-1} e=\left(-\theta_{T}\right)^{2 k} / \lambda$ and substituting for $\lambda=\left(1-\left(-\theta_{T}\right)^{2(k+1)}\right) /\left(1-\left(-\theta_{T}\right)^{2}\right)$,

$$
\begin{aligned}
\sqrt{T} e^{\prime} \widetilde{R}^{-1} \widetilde{V} & =\frac{-(-\theta)^{2 k}\left(1-\theta_{T}\right)^{2}}{\left[1-(-\theta)^{2(k+1)}\right] x+(-\theta)^{2 k}\left(1-\theta_{T}\right)^{2}} \\
& =\frac{-(-\theta)^{2 k}\left(1-\theta_{T}\right)^{2}}{\left[1-(-\theta)^{2(k+1)}\right] x}+O_{p}\left(T^{-1 / 2}\right)
\end{aligned}
$$

which converges to $-\vartheta(\kappa) / x$ setting $k=\kappa T^{1 / 2}$ and taking limit as $T \rightarrow \infty$. 
Case $K_{2 T} \leq k \leq K_{3 T}$.

Note that $\left(-\theta_{T}\right)^{k} \rightarrow 0$ if $k / \sqrt{T} \rightarrow \infty$. Let

$$
\widetilde{R}=\sigma_{e}^{2}\left[\begin{array}{ll}
1+x & e^{\prime} \\
e & I_{k}
\end{array}\right]
$$

and note that

$$
\widetilde{R}^{-1}=\sigma_{e}^{2}\left[\begin{array}{lll}
x^{-1} & -x^{-1} & 0_{1 \times k-1} \\
-x^{-1} & (1+x) x^{-1} & 0 \\
0_{k-1 \times 1} & 0 & I_{k-1}
\end{array}\right]
$$

Thus, $\|\widetilde{R}\|=O_{p}(1)$ and $\left\|\widetilde{R}^{-1}\right\|=O_{p}(1)$. ¿From Lemma A.1 (a,b,c), $\max _{K_{2 T} \leq k \leq K_{3 T}}\left\|\hat{R}_{T}-\widetilde{R}\right\|=$ $O_{p}\left(K_{3 T} / T^{1 / 2}\right)$. Also, $\max _{K_{2 T} \leq k \leq K_{3 T}}\left\|\hat{R}_{T}\right\| \leq \max _{K_{2 T} \leq k \leq K_{3 T}}\left\|\hat{R}_{T}-\widetilde{R}\right\|+\max _{K_{2 T} \leq k \leq K_{3 T}}\|\widetilde{R}\|=$ $O_{p}\left(K_{3 T} / T^{1 / 2}\right)$ since $\max _{K_{2 T} \leq k \leq K_{3 T}}\|\widetilde{R}\|=O_{p}(1)$ and $K_{3 T} / T^{1 / 2} \rightarrow \infty$. Since $\max _{K_{2 T} \leq k \leq K_{3 T}}\left\|\hat{R}_{T}^{-1}\right\| \leq$ $1 / \max _{K_{2 T} \leq k \leq K_{3 T}}\left\|\hat{R}_{T}\right\|, \max _{K_{2 T} \leq k \leq K_{3 T}}\left\|\hat{R}_{T}^{-1}\right\| \leq O_{p}\left(T^{1 / 2} / K_{3 T}\right)$. It follows that

$$
\begin{aligned}
\max _{K_{2 T} \leq k \leq K_{3 T}}\left\|\hat{R}_{T}^{-1}-\widetilde{R}^{-1}\right\| & \leq \max _{K_{2 T} \leq k \leq K_{3 T}}\left\|\hat { R } _ { T } ^ { - 1 } \left|\left\||| \hat{R}_{T}-\widetilde{R}\right\|\left\|\widetilde{R}^{-1}\right\|\right.\right. \\
& \leq O_{p}\left(T^{1 / 2} / K_{3 T}\right) O_{p}\left(K_{3 T} / T^{1 / 2}\right) O_{p}(1)=O_{p}(1) .
\end{aligned}
$$

We also have $\max _{K_{2 T} \leq k \leq K_{3 T}}\left\|\hat{V}_{T}-\tilde{V}\right\|=O_{p}\left(K_{3 T}^{1 / 2} / T^{1 / 2}\right)$ and $\|\tilde{V}\|=O_{p}(1)$. Thus,

$$
\begin{aligned}
T^{1 / 2} \hat{\beta}_{0}= & T^{1 / 2} e^{\prime} \hat{R}_{T}^{-1} \hat{V}_{T} \\
= & T^{1 / 2} e^{\prime}\left(\hat{R}_{T}^{-1}-\widetilde{R}^{-1}\right)\left(\hat{V}_{T}-\widetilde{V}\right)+T^{1 / 2} e^{\prime}\left(\hat{R}_{T}^{-1}-\widetilde{R}^{-1}\right) \widetilde{V} \\
& +T^{1 / 2} e^{\prime} \widetilde{R}^{-1}\left(\hat{V}_{T}-\widetilde{V}\right)+T^{1 / 2} e^{\prime} \widetilde{R}^{-1} \widetilde{V} \\
= & A+B+C+D .
\end{aligned}
$$

For $A$, we have

$$
\begin{aligned}
\max _{K_{2 T} \leq k \leq K_{3 T}}\left\|T^{1 / 2} e^{\prime}\left(\hat{R}_{T}^{-1}-\widetilde{R}^{-1}\right)\left(\hat{V}_{T}-\widetilde{V}\right)\right\|^{2} & \leq \max _{K_{2 T} \leq k \leq K_{3 T}} T \sum_{i=1}^{k}\left\|\hat{r}^{1, i}-\widetilde{r}^{1, i}\right\|^{2}\left\|\hat{V}_{i, T}-\widetilde{V}_{i}\right\|^{2} \\
& \leq \max _{K_{2 T} \leq k \leq K_{3 T}} c \sum_{i=1}^{k}\left\|\hat{r}^{1, i}-\widetilde{r}^{1, i}\right\|^{2} \\
& \leq \max _{K_{2 T} \leq k \leq K_{3 T}} c\left\|\hat{R}_{T}^{-1}-\widetilde{R}^{-1}\right\|^{2}=O_{p}(1) .
\end{aligned}
$$

For $B,\left\|T^{1 / 2} e^{\prime}\left(\hat{R}_{T}^{-1}-\widetilde{R}^{-1}\right) \widetilde{V}\right\|=\left\|T^{1 / 2} e^{\prime} \widetilde{R}^{-1} C \widetilde{R}^{-1} \widetilde{V}\right\|+O_{p}\left(T^{-1 / 2}\right)$ for some $\widetilde{R}$ such that $\hat{R}_{T}=\widetilde{R}+C$, where the matrix $C$ has elements that are $O_{p}\left(T^{-1 / 2}\right)$. Now

$$
\begin{aligned}
e^{\prime} \widetilde{R}^{-1} & =\left[x^{-1},-x^{-1}, 0,0,0, \ldots, 0\right], \\
\widetilde{R}^{-1} \widetilde{V} & =[0,-1,0,0, \ldots, 0]^{\prime} .
\end{aligned}
$$


Therefore $C \widetilde{R}^{-1} \widetilde{V}=-C_{.2}$ where $C_{.2}$ is the second column of the matrix $C$. Then $e^{\prime} \widetilde{R}^{-1} C \widetilde{R}^{-1} \widetilde{V}=$ $x^{-1}\left(-C_{12}+C_{22}\right)$. Since $C_{12}$ and $C_{22}=O_{p}\left(T^{-1 / 2}\right), \max _{K_{2 T} \leq k \leq K_{3 T}}\left\|T^{1 / 2} e^{\prime} \widetilde{R}^{-1} C \widetilde{R}^{-1} \widetilde{V}\right\|=O_{p}(1)$. For the third term $C$,

$$
\begin{aligned}
\max _{K_{2 T} \leq k \leq K_{3 T}}\left\|T^{1 / 2} e^{\prime} \widetilde{R}^{-1}\left(\hat{V}_{T}-\widetilde{V}\right)\right\| & =\max _{K_{2 T} \leq k \leq K_{3 T}}\left\|T^{1 / 2}\left[x^{-1},-x^{-1}, \ldots, 0\right]\left(\hat{V}_{T}-\tilde{V}\right)\right\| \\
& =\max _{K_{2 T} \leq k \leq K_{3 T}}\left\|\frac{T^{1 / 2}}{x}\left[\left(\hat{V}_{1, T}-\widetilde{V}_{1}\right)-\left(\hat{V}_{2, T}-\widetilde{V}_{2}\right)\right]\right\| \\
& \leq \frac{T^{1 / 2}}{x} \frac{c}{T^{1 / 2}}=O_{p}(1) .
\end{aligned}
$$

For $D$,

$$
T^{1 / 2} e^{\prime} \widetilde{R}^{-1} \widetilde{V}=T^{1 / 2}\left[-x^{-1}+x^{-1}\right]=0 .
$$

Combining the results, we have $T^{1 / 2} \hat{\beta}_{0}=O_{p}(1)$ uniformly in $K_{2 T} \leq k \leq K_{3 T}$.

\section{Proof of Lemma 1}

Lemma A.5 Consider the estimated autoregression (8) when the DGP is given by (14). Let $\hat{\sigma}_{k}^{2}=$ $N^{-1} \sum_{t=1}^{T} \hat{e}_{t k}^{2}$, then uniformly over $0<k \leq K_{3 T}$

$$
\ln \left(\hat{\sigma}_{k}^{2}\right)=\ln \left(\sigma_{e}^{2}\right)+\frac{\left(-\theta_{T}\right)^{2(k+1)}}{1-\left(-\theta_{T}\right)^{2(k+1)}}\left(1-\theta_{T}^{2}\right)+O_{p}\left(T^{-1 / 2}\right) .
$$

Proof: Let $E_{k}=\left(e_{k_{\max }+1 k}, e_{2 k}, \ldots, e_{T k}\right)^{\prime}$, where $e_{t k}=c T^{-1} \sum_{i=1}^{k}\left(-\theta_{T}\right)^{i} y_{t-i-1}+e_{t}-\left(-\theta_{T}\right)^{k+1} e_{t-k-1}$. Let $X=\left(X_{1}, \ldots, X_{T}\right)^{\prime}$, Then

$$
\hat{\sigma}_{k}^{2}=N^{-1} E_{k}^{\prime} E_{k}-N^{-1} E_{k}^{\prime} X\left(X^{\prime} X\right)^{-1} X^{\prime} E_{k} .
$$

By direct calculations,

$$
\begin{aligned}
N^{-1} E_{k}^{\prime} E_{k} & =N^{-1} \sum_{t=k_{\max }+1}^{T} e_{t}^{2}+\left(-\theta_{T}\right)^{2(k+1)} N^{-1} \sum_{t=k_{\max }+1}^{T} e_{t-k-1}^{2}+O_{p}\left(T^{-1 / 2}\right) \\
& =\sigma_{e}^{2}\left(1+\left(-\theta_{T}\right)^{2(k+1)}\right)+O_{p}\left(T^{-1 / 2}\right) .
\end{aligned}
$$

Consider $N^{-1} E_{k}^{\prime} X=\left(N^{-1} \sum_{t=k_{\max }+1}^{T} y_{t-1} e_{t k}, N^{-1} \sum_{t=k_{\max }+1}^{T} Z_{t-j}^{\prime} e_{t k}\right)$. Using results established in the proof of Theorem 2 ,

$$
\begin{aligned}
N^{-1} \sum_{t=k_{\max }+1}^{T} y_{t-1} e_{t k} & =N^{-1} \sum_{t=k_{\max }+1}^{T}\left(a_{T} e_{t-1}+b_{T} X_{t-1}\right)\left(e_{t}-\left(-\theta_{T}\right)^{k+1} e_{t-k-1}+\frac{c}{T} \sum_{i=1}^{k}\left(-\theta_{T}\right)^{i} y_{t-i-1}\right) \\
& =O_{p}\left(T^{-1 / 2}\right), \text { and } \\
N^{-1} \sum_{t=k_{\max }+1}^{T} z_{t-j} e_{t k} & =N^{-1} \sum_{t=k_{\max }+1}^{T}\left(e_{t-j}-\left(-\theta_{T}\right)^{k+1-j} e_{t-k-1}\right)\left(e_{t}-\left(-\theta_{T}\right)^{k+1} e_{t-k-1}\right)+O_{p}\left(T^{-1 / 2}\right)
\end{aligned}
$$




$$
\begin{aligned}
& =\left(-\theta_{T}\right)^{2(k+1)-j} N^{-1} \sum_{t=k_{\max }+1}^{T} e_{t-k-1}^{2}+O_{p}\left(T^{-1 / 2}\right), \\
& =\sigma_{e}^{2}\left(-\theta_{T}\right)^{2(k+1)-j}+O_{p}\left(T^{-1 / 2}\right) .
\end{aligned}
$$

Therefore,

$$
\begin{aligned}
N^{-1} E_{k}^{\prime} X & =\sigma_{e}^{2}\left[0, \theta_{T}^{2(k+1)-1}, \ldots,\left(-\theta_{T}\right)^{2(k+1)-k}\right]+O_{p}\left(T^{-1 / 2}\right), \\
& =\sigma_{e}^{2}\left(\theta_{T}\right)^{k+1}\left[0, \mu^{\prime}\right]+O_{p}\left(T^{-1 / 2}\right),
\end{aligned}
$$

where $\mu^{\prime}=\left[\left(-\theta_{T}\right)^{k}, \ldots,\left(-\theta_{T}\right)\right]$. From Lemma A.1,

$$
N^{-1}\left(X^{\prime} X\right)=\sigma_{e}^{2}\left[\begin{array}{lll}
1+x & e^{\prime} & \\
e & & I_{k}+\mu \mu^{\prime}
\end{array}\right]+O_{p}\left(T^{-1 / 2}\right) \equiv R+O_{p}\left(T^{-1 / 2}\right)
$$

In view of the properties of $N^{-1} E_{k}^{\prime} X$,

$$
N^{-1} E_{k}^{\prime} X\left(X^{\prime} X\right)^{-1} X^{\prime} E_{k}=\left(-\theta_{T}\right)^{2(k+1)} \mu^{\prime} R^{-1}(k) \mu+O_{p}\left(T^{-1 / 2}\right),
$$

where $R^{-1}(k)$ is the lower right $k \times k$ sub-matrix of $R^{-1}$. By partitioned matrix inversion,

$$
\begin{aligned}
R^{-1}(k) & =\left[I_{k}+\mu \mu^{\prime}-e^{\prime} e /(1+x)\right]^{-1}, \\
& =\left[J_{k}+\mu \mu^{\prime}\right]^{-1},
\end{aligned}
$$

where $J_{k}=I_{k}-e e^{\prime} /(1+x)$. For a $k \times k$ matrix $A$ and $k \times 1$ vectors $u$ and $v,\left(A+u v^{\prime}\right)^{-1}=$ $A^{-1}-A^{-1} u\left(1+v^{\prime} A^{-1} u\right)^{-1} v^{\prime} A^{-1}$. Hence, for $\lambda=1+\mu^{\prime} J_{k}^{-1} \mu$,

$$
\begin{aligned}
R^{-1}(k) & =J_{k}^{-1}-J_{k}^{-1} \mu \mu^{\prime} J_{k}^{-1} / \lambda, \\
\mu^{\prime} R^{-1}(k) \mu & =\mu^{\prime} J_{k}^{-1} \mu-\left(\mu^{\prime} J_{k}^{-1} \mu\right)^{2} / \lambda, \\
& =\frac{\mu^{\prime} J_{k}^{-1} \mu}{1+\mu^{\prime} J_{k}^{-1} \mu} .
\end{aligned}
$$

Since $J_{k}=I_{k}-e e^{\prime} /(1+x), J_{k}^{-1}=I_{k}+e e^{\prime} / x$, and

$$
\mu^{\prime} J_{k}^{-1} \mu=\mu^{\prime} \mu+\mu^{\prime} e e^{\prime} \mu / x=\mu^{\prime} \mu+\left(-\theta_{T}\right)^{2 k} / x .
$$

We also have $\mu^{\prime} \mu=\sum_{i=1}^{k}\left(-\theta_{T}\right)^{2 i}$, hence

$$
\mu^{\prime} \mu=\frac{\left(-\theta_{T}\right)^{2}-\left(-\theta_{T}\right)^{2(k+1)}}{1-\left(-\theta_{T}\right)^{2}}, 1+\mu^{\prime} \mu=\frac{1-\left(-\theta_{T}\right)^{2(k+1)}}{1-\left(-\theta_{T}\right)^{2}} .
$$

After some simplifications,

$$
\begin{aligned}
\frac{\mu^{\prime} J_{k}^{-1} \mu}{1+\mu^{\prime} J_{k}^{-1} \mu} & =\frac{\left(-\theta_{T}\right)^{2}\left[1-\left(-\theta_{T}\right)^{2 k}\right]+\left(-\theta_{T}\right)^{2 k}\left[1-\left(-\theta_{T}\right)^{2}\right] / x}{\left[1-\left(-\theta_{T}\right)^{2(k+1)}\right]+\left(-\theta_{T}\right)^{2 k}\left[1-\left(-\theta_{T}\right)^{2}\right] / x} \\
& =\left(-\theta_{T}\right)^{2}\left[\frac{1-\left(-\theta_{T}\right)^{2 k}}{1-\left(-\theta_{T}\right)^{2(k+1)}}\right]+O_{p}\left(T^{-1 / 2}\right)
\end{aligned}
$$


Hence,

$$
N^{-1} E_{k}^{\prime} X\left(X^{\prime} X\right)^{-1} X^{\prime} E_{k}=(-\theta)^{2(k+1)}\left(-\theta_{T}\right)^{2}\left[\frac{1-\left(-\theta_{T}\right)^{2 k}}{1-\left(-\theta_{T}\right)^{2(k+1)}}\right]+O_{p}\left(T^{-1 / 2}\right) .
$$

It follows that

$$
\begin{aligned}
\hat{\sigma}_{k}^{2} & \left.=\sigma_{e}^{2}\left(1+\left(-\theta_{T}\right)^{2(k+1)}-\left(-\theta_{T}\right)^{2(k+2)}\left[\frac{1-\left(-\theta_{T}\right)^{2 k}}{1-\left(-\theta_{T}\right)^{2(k+1)}}\right)\right]\right)+O_{p}\left(T^{-1 / 2}\right), \\
\ln \left(\hat{\sigma}_{k}^{2}\right) & =\ln \left(\sigma_{e}^{2}\right)+\left[\frac{(-\theta)^{2(k+1)}\left(1-\left(-\theta_{T}\right)^{2}\right)}{1-\left(-\theta_{T}\right)^{2(k+1)}}\right]+O_{p}\left(T^{-1 / 2}\right) .
\end{aligned}
$$

Lemma 1 is a consequence of Theorem 2 and Lemma A.5.

\section{Proof of Theorem 3.}

The strategy of the proof is to first determine the rate of increase of $k$ through a global minimization of the objective function. Once this rate is found, the proportionality factor is deduced. In the following, we use the notation $k \propto f(T)$ for the case where $k / f(T) \rightarrow c$ for some finite constant $c$ strictly above 0 . We also let $d \geq 0$ be a finite constant not necessarily the same throughout.

For both the $I C$ and $M I C$, it is readily seen that the objective function is $O_{p}\left(C_{T} / T^{1 / 2}\right)$ when $k / T^{1 / 2} \rightarrow \kappa(>0)$, and that it is $O_{p}\left(C_{T} k / T\right)$ when $k / T^{1 / 2} \rightarrow \infty$. Hence, irrespective of the choice of $C_{T}$, having $k / T^{1 / 2} \rightarrow \kappa$ will always, in large samples, yield a smaller value of the objective function than with $k / T^{1 / 2} \rightarrow \infty$. Hence, we only need to compare the cases $k / T^{1 / 2} \rightarrow \kappa(>0)$ and $k / T^{1 / 2} \rightarrow 0$.

We start with the analysis for the $I C$. When $k / T^{1 / 2} \rightarrow 0$, there are two cases of interest. The first is when $k^{2} C_{T} / T \rightarrow d$ in which case the objective function is $O_{p}(1 / k)$. The minimal value is obtained if $k$ is chosen as the maximal value permissible which means $k \propto \sqrt{T / C_{T}}$. The minimized objective function is then $O_{p}\left(\sqrt{C_{T}} / \sqrt{T}\right)$. The second case is when $T / k^{2} C_{T} \rightarrow d$ and the objective function is $O_{p}\left(k C_{T} / T\right)$ which is minimized by taking the minimal permissible rate of increase for $k$, again $k \propto \sqrt{T / C_{T}}$ and the minimized objective function is then $O_{p}\left(\sqrt{C_{T}} / \sqrt{T}\right)$. So the case with $k / T^{1 / 2} \rightarrow \kappa(>0)$ yields an objective function of order $O_{p}\left(C_{T} / T^{1 / 2}\right)$ and the case $k / T^{1 / 2} \rightarrow 0$ an objective function of order $O_{p}\left(\sqrt{C_{T}} / \sqrt{T}\right)$. Hence, $k \propto \sqrt{T / C_{T}}$. To derive the constant of proportionality, note that if $C_{T}$ is fixed, $k=\kappa \sqrt{T}$ and the objective function is (ignoring constants)

$$
\frac{2 \delta}{\sqrt{T}} \vartheta(\kappa)+\frac{C_{T}}{\sqrt{T}} \kappa
$$

Taking derivatives and equating to zero yields the stated solution. When $C_{T} \rightarrow \infty$ as $T \rightarrow \infty$, the objective function (ignoring constants) is

$$
\frac{1}{\kappa \sqrt{T / C_{T}}}+\kappa \frac{C_{T} \sqrt{T / C_{T}}}{T}
$$


and the value of $\kappa$ that minimizes this expression is 1 . Consider now the $M I C(k)$. The first case is where

$$
\frac{C_{T}}{k} \rightarrow d \text { and } \frac{C_{T} k^{2}}{T} \rightarrow d
$$

Then the objective function when $k / T^{1 / 2} \rightarrow 0$ is $O_{p}\left(k^{-1}\right)$. Hence, the highest possible rate is chosen and is given by $k \propto \sqrt{T / C_{T}}$ and the minimized objective function is of order $O_{p}\left(\sqrt{C_{T} / T}\right)$. Since the objective function when $k / T^{1 / 2} \rightarrow \kappa(>0)$ is $O_{p}\left(C_{T} / T^{1 / 2}\right)$, we have $k \propto T^{1 / 2}$ if $C_{T}$ is fixed and $k \propto \sqrt{T / C_{T}}$ if $C_{T} \rightarrow \infty$. However, in view of the requirement that $C_{T} / k \rightarrow d$ this implies the need for $C_{T}^{3} / T \rightarrow d$. To obtain the constant of proportionality when $C_{T}$ is fixed, we substitute $k=\kappa \sqrt{T}$ in (16) and equate its first derivative of (16) to zero and solve for $\kappa$. When $C_{T}$ is increasing, we substitute $k=\kappa \sqrt{T / C_{T}}$ in (15), and again equate the first derivative to zero and solve for $\kappa$.

Consider now the case where $C_{T}^{3} / T \rightarrow \infty$. There are two cases to cover when either of the conditions in (A.8) is not satisfied. Suppose first that $k^{3} / T \rightarrow d$ in which case $k / C_{T} \rightarrow d$. The objective function in the case $k / T^{1 / 2} \rightarrow 0$ is $O_{p}\left(C_{T} / k^{2}\right)$ and the highest rate for $k$ is chosen, namely $k \propto T^{1 / 3}$. The minimized objective function is then of order $O_{p}\left(C_{T} / T^{2 / 3}\right)$ which is of a smaller order than the case with $k / T^{1 / 2} \rightarrow \kappa(>0)$. Hence, $k \propto T^{1 / 3}$ is the resulting rate. If $T / k^{3} \rightarrow d$, in which case $C_{T} k^{2} / T \rightarrow \infty$, the objective function is of order $O_{p}\left(k C_{T} / T\right)$ and the smallest rate for $k$ is optimal. So again, $k \propto T^{1 / 3}$ and the minimized objective function is also of order $O_{p}\left(C_{T} / T^{2 / 3}\right)$. To obtain the factor of proportionality, let $k=\kappa T^{1 / 3}$. The objective function is then (ignoring constants)

$$
\frac{1}{\kappa T^{1 / 3}}+\frac{C_{T} Z_{1}}{\kappa^{2} T^{2 / 3}}+\frac{C_{T} \kappa}{T^{2 / 3}}
$$

When $C_{T}^{3} / T \rightarrow \infty$, the first term is negligible compared to the last two. Hence, minimizing the last two terms with respect to $\kappa$ yields $\kappa=\left(2 Z_{1}\right)^{1 / 3}$. 
Table 1: Asymptotic Critical alues

\begin{tabular}{|c|c|c|c|c|c|}
\hline & $M$ & $M_{t}$ & & $M S$ & ${ }_{T} \quad M_{T}$ \\
\hline & \multicolumn{5}{|c|}{$\begin{array}{lll}\mathrm{a} & p=0 & \bar{c}=-7.0\end{array}$} \\
\hline 01 & 138 & 258 & & 174 & 178 \\
\hline 05 & 81 & 18 & & 233 & 317 \\
\hline \multirow[t]{2}{*}{10} & 57 & 162 & & 275 & 445 \\
\hline & \multicolumn{5}{|c|}{$p=1 \bar{c}=-13.5$} \\
\hline 01 & 238 & 342 & & 143 & 403 \\
\hline 05 & 173 & $\begin{array}{ll}2 & 1\end{array}$ & & 168 & 548 \\
\hline 10 & 142 & 262 & & 185 & 667 \\
\hline
\end{tabular}

For $M Z_{\alpha}^{G L S}, M Z^{G L S}$ and $A D F^{G L S}$ with $p=0$, the critical values are from Fuller (1976). The other entries were obtained using 20,000 simulations and 5,000 steps to approximate the Wiener processes. 
Table 2.a: Exact Size of the Tests at Selected alues of $k: p=0$.

\begin{tabular}{|c|c|c|c|c|c|c|c|c|c|c|c|c|c|}
\hline$M A$ Case & $T$ & $\theta$ & $k=0$ & 1 & 2 & 3 & 4 & 5 & 6 & 7 & 8 & 9 & 10 \\
\hline \multirow{10}{*}{$\bar{Z}_{\alpha}^{G L S}$} & 100 & -0.8 & 0.994 & 0.904 & 0.808 & 0.740 & 0.698 & 0.672 & 0.654 & 0.644 & 0.636 & 0.633 & 0.627 \\
\hline & & -0.5 & 0.618 & 0.322 & 0.224 & 0.193 & 0.176 & 0.181 & 0.182 & 0.192 & 0.197 & 0.207 & 0.216 \\
\hline & & 0.0 & 0.072 & 0.082 & 0.089 & 0.104 & 0.112 & 0.125 & 0.137 & 0.152 & 0.164 & 0.179 & 0.190 \\
\hline & & 0.5 & 0.010 & 0.143 & 0.056 & 0.116 & 0.100 & 0.129 & 0.135 & 0.153 & 0.158 & 0.179 & 0.187 \\
\hline & & 0.8 & 0.006 & 0.172 & 0.036 & 0.147 & 0.071 & 0.155 & 0.108 & 0.166 & 0.138 & 0.188 & 0.169 \\
\hline & 250 & -0.8 & 0.987 & 0.861 & 0.725 & 0.641 & 0.592 & 0.559 & 0.539 & 0.524 & 0.515 & 0.507 & 0.504 \\
\hline & & -0.5 & 0.553 & 0.258 & 0.166 & 0.124 & 0.112 & 0.110 & 0.106 & 0.112 & 0.111 & 0.115 & 0.116 \\
\hline & & 0.0 & 0.060 & 0.062 & 0.063 & 0.066 & 0.069 & 0.075 & 0.077 & 0.081 & 0.087 & 0.087 & 0.092 \\
\hline & & 0.5 & 0.008 & 0.111 & 0.047 & 0.080 & 0.065 & 0.077 & 0.076 & 0.083 & 0.084 & 0.091 & 0.095 \\
\hline & & 0.8 & 0.004 & 0.145 & 0.030 & 0.108 & 0.049 & 0.099 & 0.062 & 0.096 & 0.074 & 0.100 & 0.085 \\
\hline \multirow{10}{*}{$\bar{M} Z_{\alpha}^{G L S}$} & 100 & -0.8 & 0.990 & 0.828 & 0.543 & 0.319 & 0.187 & 0.129 & 0.095 & 0.086 & 0.079 & 0.077 & 0.078 \\
\hline & & -0.5 & 0.595 & 0.241 & 0.126 & 0.097 & 0.084 & 0.092 & 0.097 & 0.106 & 0.120 & 0.132 & 0.141 \\
\hline & & 0.0 & 0.065 & 0.070 & 0.082 & 0.095 & 0.104 & 0.119 & 0.130 & 0.145 & 0.158 & 0.172 & 0.185 \\
\hline & & 0.5 & 0.008 & 0.139 & 0.054 & 0.113 & 0.097 & 0.126 & 0.132 & 0.150 & 0.156 & 0.176 & 0.186 \\
\hline & & 0.8 & 0.004 & 0.168 & 0.035 & 0.143 & 0.068 & 0.153 & 0.106 & 0.164 & 0.136 & 0.186 & 0.168 \\
\hline & 250 & -0.8 & 0.987 & 0.828 & 0.597 & 0.407 & 0.260 & 0.166 & 0.107 & 0.075 & 0.055 & 0.044 & 0.035 \\
\hline & & -0.5 & 0.545 & 0.227 & 0.121 & 0.081 & 0.067 & 0.063 & 0.062 & 0.066 & 0.070 & 0.071 & 0.074 \\
\hline & & 0.0 & 0.058 & 0.059 & 0.061 & 0.064 & 0.066 & 0.072 & 0.076 & 0.079 & 0.084 & 0.084 & 0.088 \\
\hline & & 0.5 & 0.008 & 0.110 & 0.045 & 0.078 & 0.064 & 0.076 & 0.075 & 0.082 & 0.083 & 0.090 & 0.095 \\
\hline & & 0.8 & 0.004 & 0.145 & 0.029 & 0.108 & 0.048 & 0.099 & 0.061 & 0.095 & 0.074 & 0.100 & 0.084 \\
\hline \multirow{10}{*}{$D F^{G L S}$} & 100 & -0.8 & 0.991 & 0.843 & 0.624 & 0.455 & 0.322 & 0.243 & 0.187 & 0.157 & 0.129 & 0.114 & 0.104 \\
\hline & & -0.5 & 0.605 & 0.263 & 0.147 & 0.108 & 0.089 & 0.087 & 0.081 & 0.079 & 0.076 & 0.078 & 0.075 \\
\hline & & 0.0 & 0.073 & 0.070 & 0.072 & 0.074 & 0.072 & 0.072 & 0.070 & 0.070 & 0.071 & 0.074 & 0.073 \\
\hline & & 0.5 & 0.010 & 0.124 & 0.046 & 0.081 & 0.063 & 0.075 & 0.074 & 0.077 & 0.078 & 0.075 & 0.071 \\
\hline & & 0.8 & 0.005 & 0.150 & 0.030 & 0.107 & 0.043 & 0.089 & 0.054 & 0.082 & 0.059 & 0.080 & 0.064 \\
\hline & 250 & -0.8 & 0.985 & 0.823 & 0.613 & 0.449 & 0.332 & 0.247 & 0.194 & 0.155 & 0.128 & 0.109 & 0.099 \\
\hline & & -0.5 & 0.535 & 0.227 & 0.123 & 0.084 & 0.068 & 0.064 & 0.062 & 0.062 & 0.061 & 0.058 & 0.060 \\
\hline & & 0.0 & 0.057 & 0.054 & 0.054 & 0.053 & 0.056 & 0.055 & 0.058 & 0.058 & 0.056 & 0.055 & 0.054 \\
\hline & & 0.5 & 0.009 & 0.103 & 0.043 & 0.070 & 0.055 & 0.061 & 0.057 & 0.058 & 0.057 & 0.060 & 0.060 \\
\hline & & 0.8 & 0.004 & 0.133 & 0.027 & 0.090 & 0.039 & 0.074 & 0.046 & 0.068 & 0.049 & 0.065 & 0.051 \\
\hline$A R$ Case & $T$ & $\rho$ & $k=0$ & 1 & 2 & 3 & 4 & 5 & 6 & 7 & 8 & 9 & 10 \\
\hline \multirow{4}{*}{$\bar{Z}_{\alpha}^{G L S}$} & 100 & -0.8 & 0.802 & 0.286 & 0.286 & 0.295 & 0.300 & 0.308 & 0.314 & 0.322 & 0.328 & 0.340 & 0.346 \\
\hline & & 0.8 & 0.000 & 0.102 & 0.115 & 0.135 & 0.143 & 0.158 & 0.172 & 0.191 & 0.201 & 0.217 & 0.226 \\
\hline & 250 & -0.8 & 0.737 & 0.170 & 0.171 & 0.171 & 0.173 & 0.176 & 0.179 & 0.182 & 0.185 & 0.184 & 0.183 \\
\hline & & 0.8 & 0.000 & 0.069 & 0.071 & 0.077 & 0.082 & 0.084 & 0.090 & 0.094 & 0.093 & 0.099 & 0.100 \\
\hline \multirow{4}{*}{$\bar{M} Z_{\alpha}^{G L S}$} & 100 & -0.8 & 0.784 & 0.027 & 0.035 & 0.050 & 0.065 & 0.081 & 0.089 & 0.113 & 0.120 & 0.140 & 0.149 \\
\hline & & 0.8 & 0.000 & 0.100 & 0.114 & 0.134 & 0.142 & 0.157 & 0.170 & 0.190 & 0.199 & 0.216 & 0.225 \\
\hline & 250 & -0.8 & 0.730 & 0.043 & 0.041 & 0.047 & 0.048 & 0.050 & 0.058 & 0.062 & 0.064 & 0.071 & 0.071 \\
\hline & & 0.8 & 0.000 & 0.069 & 0.071 & 0.077 & 0.082 & 0.084 & 0.090 & 0.094 & 0.092 & 0.099 & 0.100 \\
\hline \multirow{4}{*}{$D F^{G L S}$} & 100 & -0.8 & 0.791 & 0.073 & 0.074 & 0.077 & 0.079 & 0.080 & 0.076 & 0.081 & 0.075 & 0.083 & 0.078 \\
\hline & & 0.8 & 0.000 & 0.066 & 0.070 & 0.069 & 0.068 & 0.069 & 0.071 & 0.072 & 0.071 & 0.077 & 0.079 \\
\hline & 250 & -0.8 & 0.718 & 0.057 & 0.054 & 0.056 & 0.054 & 0.055 & 0.057 & 0.057 & 0.057 & 0.059 & 0.058 \\
\hline & & 0.8 & 0.000 & 0.059 & 0.061 & 0.063 & 0.062 & 0.060 & 0.060 & 0.060 & 0.058 & 0.058 & 0.054 \\
\hline
\end{tabular}


Table 2.b: Exact Size of of the Tests at Selected alues of $k: p=1$.

\begin{tabular}{|c|c|c|c|c|c|c|c|c|c|c|c|c|c|}
\hline$M A$ Case & $T$ & $\theta$ & $k=0$ & 1 & 2 & 3 & 4 & 5 & 6 & 7 & 8 & 9 & 10 \\
\hline \multirow{10}{*}{$\bar{Z}_{\alpha}^{G L S}$} & 100 & -0.8 & 1.000 & 0.994 & 0.965 & 0.940 & 0.918 & 0.906 & 0.893 & 0.888 & 0.882 & 0.882 & 0.883 \\
\hline & & -0.5 & 0.810 & 0.470 & 0.340 & 0.299 & 0.290 & 0.309 & 0.323 & 0.358 & 0.376 & 0.413 & 0.450 \\
\hline & & 0.0 & 0.053 & 0.076 & 0.100 & 0.136 & 0.169 & 0.222 & 0.259 & 0.313 & 0.346 & 0.400 & 0.430 \\
\hline & & 0.5 & 0.001 & 0.169 & 0.059 & 0.163 & 0.155 & 0.226 & 0.253 & 0.303 & 0.343 & 0.397 & 0.432 \\
\hline & & 0.8 & 0.001 & 0.219 & 0.023 & 0.229 & 0.091 & 0.284 & 0.190 & 0.350 & 0.297 & 0.422 & 0.389 \\
\hline & 250 & -0.8 & 1.000 & 0.992 & 0.960 & 0.927 & 0.899 & 0.875 & 0.859 & 0.848 & 0.839 & 0.833 & 0.830 \\
\hline & & -0.5 & 0.801 & 0.404 & 0.253 & 0.202 & 0.181 & 0.174 & 0.176 & 0.179 & 0.188 & 0.199 & 0.209 \\
\hline & & 0.0 & 0.047 & 0.057 & 0.064 & 0.077 & 0.081 & 0.094 & 0.104 & 0.113 & 0.129 & 0.142 & 0.154 \\
\hline & & 0.5 & 0.002 & 0.127 & 0.037 & 0.092 & 0.071 & 0.097 & 0.095 & 0.120 & 0.122 & 0.143 & 0.153 \\
\hline & & 0.8 & 0.001 & 0.176 & 0.014 & 0.129 & 0.038 & 0.131 & 0.068 & 0.139 & 0.099 & 0.162 & 0.136 \\
\hline \multirow{10}{*}{$\bar{M} Z_{\alpha}^{G L S}$} & 100 & -0.8 & 1.000 & 0.925 & 0.592 & 0.345 & $\overline{0.220}$ & 0.185 & 0.159 & $\overline{0.163}$ & $\overline{0.163}$ & 0.185 & 0.183 \\
\hline & & -0.5 & 0.750 & 0.252 & 0.104 & 0.093 & 0.099 & 0.132 & 0.159 & 0.204 & 0.234 & 0.285 & 0.317 \\
\hline & & 0.0 & 0.032 & 0.055 & 0.080 & 0.115 & 0.152 & 0.206 & 0.243 & 0.300 & 0.332 & 0.390 & 0.419 \\
\hline & & 0.5 & 0.001 & 0.161 & 0.054 & 0.155 & 0.148 & 0.219 & 0.246 & 0.299 & 0.338 & 0.392 & 0.427 \\
\hline & & 0.8 & 0.001 & 0.211 & 0.021 & 0.224 & 0.087 & 0.277 & 0.186 & 0.346 & 0.294 & 0.417 & 0.386 \\
\hline & 250 & -0.8 & 1.000 & 0.978 & 0.800 & 0.514 & 0.276 & 0.147 & 0.087 & 0.058 & 0.048 & 0.040 & 0.036 \\
\hline & & -0.5 & 0.786 & 0.311 & 0.126 & 0.074 & 0.057 & 0.059 & 0.065 & 0.069 & 0.082 & 0.100 & 0.106 \\
\hline & & 0.0 & 0.040 & 0.049 & 0.057 & 0.070 & 0.077 & 0.088 & 0.099 & 0.108 & 0.124 & 0.136 & 0.149 \\
\hline & & 0.5 & 0.002 & 0.124 & 0.034 & 0.090 & 0.069 & 0.095 & 0.094 & 0.117 & 0.121 & 0.142 & 0.150 \\
\hline & & 0.8 & 0.001 & 0.174 & 0.014 & 0.128 & 0.036 & 0.130 & 0.066 & 0.137 & 0.098 & 0.160 & 0.135 \\
\hline \multirow{10}{*}{$D F^{G L S}$} & 100 & -0.8 & $\begin{array}{l}1.000 \\
\end{array}$ & 0.977 & $\overline{0.815}$ & $\overline{0.600}$ & 0.415 & $\overline{0.293}$ & $\overline{c 0.203}$ & 0.167 & 0.125 & $\overline{0.116}$ & $\overline{0.098}$ \\
\hline & & -0.5 & 0.838 & 0.375 & 0.176 & 0.112 & 0.087 & 0.084 & 0.074 & 0.070 & 0.068 & 0.069 & 0.065 \\
\hline & & 0.0 & 0.070 & 0.072 & 0.067 & 0.072 & 0.069 & 0.073 & 0.072 & 0.071 & 0.069 & 0.068 & 0.061 \\
\hline & & 0.5 & 0.003 & 0.160 & 0.045 & 0.091 & 0.064 & 0.073 & 0.062 & 0.066 & 0.061 & 0.067 & 0.062 \\
\hline & & 0.8 & 0.002 & 0.209 & 0.021 & 0.127 & 0.036 & 0.099 & 0.046 & 0.085 & 0.053 & 0.076 & 0.061 \\
\hline & 250 & $\begin{array}{l}-0.8 \\
\end{array}$ & 1.000 & 0.984 & 0.875 & 0.685 & 0.504 & 0.362 & 0.259 & 0.194 & 0.150 & 0.120 & 0.099 \\
\hline & & -0.5 & 0.809 & 0.352 & 0.161 & 0.101 & 0.072 & 0.061 & 0.059 & 0.053 & 0.053 & 0.056 & 0.052 \\
\hline & & 0.0 & 0.053 & 0.055 & 0.055 & 0.056 & 0.056 & 0.054 & 0.054 & 0.054 & 0.055 & 0.055 & 0.054 \\
\hline & & 0.5 & 0.002 & 0.126 & 0.034 & 0.071 & 0.048 & 0.057 & 0.053 & 0.054 & 0.052 & 0.056 & 0.050 \\
\hline & & 0.8 & 0.001 & 0.172 & 0.015 & 0.098 & 0.024 & 0.075 & 0.032 & 0.063 & 0.038 & 0.058 & 0.042 \\
\hline$A R$ Case & $\bar{T}$ & $\rho$ & $k=0$ & 1 & 2 & 3 & 4 & 5 & 6 & 7 & 8 & 9 & 10 \\
\hline \multirow{4}{*}{$\bar{Z}_{\alpha}^{G L S}$} & 100 & -0.8 & 0.960 & 0.497 & 0.509 & 0.526 & 0.549 & 0.574 & 0.593 & 0.616 & 0.640 & 0.669 & 0.690 \\
\hline & & 0.8 & 0.000 & 0.123 & 0.162 & 0.213 & 0.252 & 0.312 & 0.351 & 0.410 & 0.441 & 0.489 & 0.514 \\
\hline & 250 & $\begin{array}{l}-0.8 \\
\end{array}$ & 0.956 & 0.319 & 0.327 & 0.330 & 0.335 & 0.345 & 0.352 & 0.360 & 0.369 & 0.375 & 0.379 \\
\hline & & 0.8 & 0.000 & 0.070 & 0.077 & 0.089 & 0.099 & 0.119 & 0.129 & 0.150 & 0.159 & 0.176 & 0.183 \\
\hline \multirow{4}{*}{$\bar{M} Z_{\alpha}^{G L S}$} & 100 & $\begin{array}{l}-0.8 \\
\end{array}$ & 0.941 & 0.001 & 0.008 & 0.030 & 0.058 & 0.104 & 0.140 & 0.191 & 0.233 & 0.290 & 0.329 \\
\hline & & 0.8 & 0.000 & 0.121 & 0.160 & 0.211 & 0.249 & 0.309 & 0.349 & 0.408 & 0.439 & 0.487 & 0.513 \\
\hline & 250 & -0.8 & 0.952 & 0.011 & 0.015 & 0.020 & 0.028 & 0.037 & 0.050 & 0.062 & 0.076 & 0.092 & 0.106 \\
\hline & & 0.8 & 0.000 & 0.070 & 0.076 & 0.089 & 0.099 & 0.119 & 0.128 & 0.150 & 0.159 & 0.175 & 0.183 \\
\hline \multirow{4}{*}{$D F^{G L S}$} & 100 & $\begin{array}{l}-0.8 \\
\end{array}$ & 0.967 & $\begin{array}{c}0.069 \\
\end{array}$ & 0.067 & 0.067 & $\overline{0.060}$ & 20.068 & 0.059 & 0.065 & $\overline{0.063}$ & 0.065 & 0.065 \\
\hline & & 0.8 & 0.000 & 0.072 & 0.067 & 0.070 & 0.071 & 0.069 & 0.069 & 0.074 & 0.066 & 0.072 & 0.071 \\
\hline & 250 & -0.8 & 0.959 & 0.052 & 0.051 & 0.052 & 0.050 & 0.049 & 0.050 & 0.050 & 0.051 & 0.052 & 0.053 \\
\hline & & 0.8 & 0.000 & 0.057 & 0.055 & 0.057 & 0.054 & 0.055 & 0.058 & 0.056 & 0.055 & 0.059 & 0.058 \\
\hline
\end{tabular}


Table 3.a: alues of of the proportionalinity factor $\kappa$

\begin{tabular}{|c|c|c|c|c|c|c|c|c|c|c|c|c|c|}
\hline & & \multicolumn{6}{|c|}{$p=0$} & \multicolumn{6}{|c|}{$p=1$} \\
\hline$\delta$ & & 0.50 & 1.00 & 1.50 & 2.00 & 5.00 & 8.00 & 0.50 & 1.00 & 1.50 & 2.00 & 5.00 & 8.00 \\
\hline \multirow[t]{4}{*}{100} & $b i c$ & 0.99 & 0.96 & 0.93 & 0.89 & 0.67 & 0.54 & 0.99 & 0.96 & 0.93 & 0.89 & 0.67 & 0.54 \\
\hline & aic & 0.69 & 0.65 & 0.61 & 0.57 & 0.39 & 0.30 & 0.69 & 0.65 & 0.61 & 0.57 & 0.39 & 0.30 \\
\hline & mbic & 3.59 & 1.73 & 1.24 & 1.04 & 0.68 & 0.54 & 6.34 & 2.89 & 1.87 & 0.40 & 0.71 & 0.54 \\
\hline & maic & 1.70 & 0.88 & 0.68 & 0.59 & 0.39 & 0.30 & 2.96 & 1.37 & 0.90 & 0.70 & 0.39 & 0.30 \\
\hline \multirow[t]{4}{*}{150} & bic & 0.99 & 0.96 & 0.93 & 0.90 & 0.69 & 0.55 & 0.99 & 0.96 & 0.93 & 0.90 & 0.69 & 0.55 \\
\hline & aic & 0.69 & 0.65 & 0.61 & 0.57 & 0.39 & 0.30 & 0.69 & 0.65 & 0.61 & 0.57 & 0.39 & 0.30 \\
\hline & mbic & 3.58 & 1.73 & 1.24 & 1.04 & 0.69 & 0.55 & 6.41 & 2.92 & 1.88 & 1.42 & 0.72 & 0.56 \\
\hline & maic & 1.63 & 0.85 & 0.67 & 0.59 & 0.39 & 0.30 & 2.87 & 1.32 & 0.88 & 0.69 & 0.39 & 0.30 \\
\hline \multirow[t]{4}{*}{250} & bic & 0.99 & 0.97 & 0.94 & 0.90 & 0.70 & 0.57 & 0.99 & 0.67 & 0.94 & 0.90 & 0.70 & 0.57 \\
\hline & aic & 0.69 & 0.65 & 0.61 & 0.57 & 0.39 & 0.30 & 0.69 & 0.65 & 0.61 & 0.57 & 0.39 & 0.30 \\
\hline & mbic & 3.55 & 1.71 & 1.24 & 1.04 & 0.71 & 0.57 & 6.46 & 2.93 & 1.89 & 1.42 & 0.73 & 0.57 \\
\hline & maic & 1.55 & 0.82 & 0.66 & 0.58 & 0.39 & 0.30 & 2.75 & 1.27 & 0.85 & 0.67 & 0.39 & 0.30 \\
\hline \multirow[t]{4}{*}{500} & bic & 0.99 & 0.97 & 0.94 & 0.91 & 0.72 & 0.58 & 0.99 & 0.97 & 0.94 & 0.91 & 0.72 & 0.58 \\
\hline & aic & 0.69 & 0.65 & 0.61 & 0.57 & 0.39 & 0.30 & 0.69 & 0.65 & 0.61 & 0.57 & 0.39 & 0.30 \\
\hline & mbic & 3.47 & 1.68 & 1.23 & 1.04 & 0.72 & 0.58 & 6.46 & 2.92 & 1.89 & 1.42 & 0.75 & 0.59 \\
\hline & maic & 1.44 & 0.79 & 0.65 & 0.58 & 0.39 & 0.30 & 2.60 & 1.20 & 0.81 & 0.65 & 0.39 & 0.30 \\
\hline \multirow[t]{4}{*}{5000} & bic & 0.99 & 0.98 & 0.96 & 0.93 & 0.76 & 0.63 & 0.99 & 0.98 & 0.96 & 0.93 & 0.76 & 0.63 \\
\hline & aic & 0.69 & 0.65 & 0.61 & 0.57 & 0.39 & 0.30 & 0.69 & 0.65 & 0.61 & 0.57 & 0.39 & 0.30 \\
\hline & mbic & 3.06 & 1.52 & 1.16 & 1.02 & 0.76 & 0.63 & 6.12 & 2.74 & 1.78 & 1.36 & 0.78 & 0.63 \\
\hline & maic & 1.14 & 0.71 & 0.62 & 0.57 & 0.39 & 0.30 & 2.12 & 1.00 & 0.72 & 0.60 & 0.39 & 0.30 \\
\hline \multirow[t]{4}{*}{50000} & bic & 0.99 & 0.98 & 0.96 & 0.94 & 0.79 & 0.66 & 0.99 & 0.98 & 0.96 & 0.94 & 0.79 & 0.66 \\
\hline & aic & 0.69 & 0.65 & 0.61 & 0.57 & 0.39 & 0.30 & 0.69 & 0.65 & 0.61 & 0.57 & 0.39 & 0.30 \\
\hline & mbic & 2.56 & 1.34 & 1.08 & 0.99 & 0.79 & 0.67 & 5.43 & 2.42 & 1.59 & 1.25 & 0.80 & 0.66 \\
\hline & maic & 0.92 & 0.67 & 0.61 & 0.57 & 0.39 & 0.30 & 1.69 & 0.85 & 0.66 & 0.58 & 0.39 & 0.30 \\
\hline
\end{tabular}


Table 3.b: $k$ Chosen by inimizing the Asymptotic Approximation of the $\quad$ IC and IC 18

\begin{tabular}{|c|c|cccccc|cccccc|}
\hline \hline & & \multicolumn{7}{|c|}{$p=0$} & \multicolumn{7}{c|}{$p=1$} \\
\hline$T \quad \delta$ & & 0.50 & 1.00 & 1.50 & 2.00 & 5.00 & 8.00 & 0.50 & 1.00 & 1.50 & 2.00 & 5.00 & 8.00 \\
\hline 100 & bic & 4 & 4 & 4 & 4 & 3 & 2 & 4 & 4 & 4 & 4 & 3 & 2 \\
& aic & 6 & 6 & 6 & 5 & 3 & 3 & 6 & 6 & 6 & 5 & 3 & 3 \\
& mbic & 16 & 8 & 5 & 4 & 3 & 2 & 29 & 13 & 8 & 6 & 3 & 2 \\
& maic & 17 & 8 & 6 & 5 & 3 & 3 & 29 & 13 & 9 & 7 & 3 & 3 \\
\hline 150 & bic & 5 & 5 & 5 & 4 & 3 & 3 & 5 & 5 & 5 & 4 & 3 & 3 \\
& aic & 8 & 8 & 7 & 7 & 4 & 3 & 8 & 8 & 7 & 7 & 4 & 3 \\
& mbic & 19 & 9 & 6 & 5 & 3 & 3 & 35 & 15 & 10 & 7 & 3 & 3 \\
& maic & 20 & 10 & 8 & 7 & 4 & 3 & 35 & 16 & 10 & 8 & 4 & 3 \\
\hline 250 & bic & 6 & 6 & 6 & 6 & 4 & 3 & 6 & 6 & 6 & 6 & 4 & 3 \\
& aic & 10 & 10 & 9 & 9 & 6 & 4 & 10 & 10 & 9 & 9 & 6 & 4 \\
& mbic & 23 & 11 & 8 & 7 & 4 & 3 & 43 & 19 & 12 & 9 & 4 & 3 \\
& maic & 24 & 13 & 10 & 9 & 6 & 4 & 43 & 20 & 13 & 10 & 6 & 4 \\
\hline 500 & bic & 8 & 8 & 8 & 8 & 6 & 5 & 8 & 8 & 8 & 8 & 6 & 5 \\
& aic & 15 & 14 & 13 & 12 & 8 & 6 & 15 & 14 & 13 & 12 & 8 & 6 \\
& mbic & 31 & 15 & 11 & 9 & 6 & 5 & 57 & 26 & 16 & 12 & 6 & 5 \\
& maic & 32 & 17 & 14 & 13 & 8 & 6 & 58 & 27 & 18 & 14 & 8 & 6 \\
\hline 5000 & bic & 24 & 23 & 23 & 22 & 18 & 15 & 24 & 23 & 23 & 22 & 18 & 15 \\
& aic & 49 & 46 & 43 & 40 & 27 & 21 & 49 & 46 & 43 & 40 & 27 & 21 \\
& mbic & 74 & 37 & 28 & 24 & 18 & 15 & 148 & 66 & 43 & 33 & 19 & 15 \\
& maic & 80 & 50 & 44 & 40 & 27 & 21 & 150 & 71 & 50 & 42 & 27 & 21 \\
\hline 50000 & bic & 67 & 66 & 65 & 63 & 53 & 45 & 67 & 66 & 65 & 63 & 53 & 45 \\
& aic & 154 & 147 & 137 & 128 & 88 & 68 & 154 & 147 & 137 & 128 & 88 & 68 \\
& mbic & 174 & 91 & 74 & 67 & 54 & 45 & 369 & 164 & 108 & 85 & 54 & 45 \\
& maic & 207 & 151 & 138 & 128 & 88 & 68 & 379 & 190 & 147 & 130 & 88 & 68 \\
\hline \hline \multirow{5}{*}{50}
\end{tabular}


Table 4.a: Selected $k$ for $p=0$.

\begin{tabular}{|c|c|c|c|c|c|c|c|c|c|c|c|c|}
\hline & \multicolumn{12}{|c|}{ MA case: $v=(1+\theta L) e$} \\
\hline $\mathrm{T}$ & \multicolumn{6}{|c|}{100} & \multicolumn{6}{|c|}{250} \\
\hline$\theta$ & $k^{*}$ & $k^{*}$ & $k$ & $k$ & $k$ & $k$ & $k^{*}$ & $k^{*}$ & $k$ & $k$ & $k$ & $k$ \\
\hline-0.8 & 8 & 8 & 2 & 4 & 5 & 6 & 7 & 10 & 4 & 6 & 5 & 7 \\
\hline-0.7 & 6 & 10 & 2 & 3 & 3 & 4 & 6 & 7 & 3 & 5 & 4 & 5 \\
\hline-0.6 & 5 & 7 & 1 & 2 & 2 & 3 & 5 & 6 & 2 & 4 & 3 & 4 \\
\hline-0.5 & 3 & 7 & 1 & 2 & 2 & 2 & 4 & 4 & 2 & 3 & 2 & 3 \\
\hline-0.4 & 2 & 4 & 1 & 2 & 1 & 2 & 2 & 2 & 1 & 2 & 2 & 2 \\
\hline-0.3 & 2 & 3 & 1 & 1 & 1 & 1 & 2 & 2 & 1 & 2 & 1 & 2 \\
\hline-0.2 & 1 & 2 & 0 & 1 & 1 & 1 & 1 & 1 & 1 & 1 & 1 & 1 \\
\hline-0.1 & 1 & 1 & 0 & 0 & 0 & 0 & 1 & 1 & 0 & 1 & 0 & 1 \\
\hline 0.0 & 0 & 0 & 0 & 0 & 0 & 0 & 0 & 0 & 0 & 0 & 0 & 0 \\
\hline 0.1 & 0 & 0 & 0 & 0 & 0 & 0 & 0 & 0 & 0 & 1 & 0 & 1 \\
\hline 0.2 & 0 & 0 & 0 & 1 & 0 & 1 & 1 & 1 & 1 & 1 & 1 & 1 \\
\hline 0.3 & 2 & 2 & 1 & 1 & 1 & 1 & 2 & 1 & 1 & 2 & 1 & 2 \\
\hline 0.4 & 2 & 2 & 1 & 2 & 1 & 2 & 2 & 2 & 1 & 2 & 2 & 2 \\
\hline 0.5 & 2 & 2 & 1 & 2 & 2 & 2 & 2 & 2 & 2 & 3 & 2 & 3 \\
\hline 0.6 & 2 & 2 & 2 & 3 & 2 & 3 & 2 & 2 & 2 & 4 & 2 & 4 \\
\hline 0.7 & 2 & 2 & 2 & 4 & 2 & 4 & 2 & 2 & 3 & 5 & 3 & 5 \\
\hline \multirow[t]{3}{*}{0.8} & 2 & 2 & 3 & 5 & 3 & 5 & 2 & 2 & 4 & 7 & 4 & 7 \\
\hline & \multicolumn{12}{|c|}{ AR case: $(1-\rho L) v=e$} \\
\hline & \multicolumn{6}{|c|}{100} & \multicolumn{6}{|c|}{250} \\
\hline$\rho$ & $k^{*}$ & $k^{*}$ & $k$ & $k$ & $k$ & $k$ & $k^{*}$ & $k^{*}$ & $k$ & $k$ & $k$ & $k$ \\
\hline-0.8 & 1 & 1 & 1 & 1 & 1 & 1 & 1 & 1 & 1 & 1 & 1 & 1 \\
\hline-0.7 & 1 & 1 & 1 & 1 & 1 & 1 & 1 & 1 & 1 & 1 & 1 & 1 \\
\hline-0.6 & 1 & 1 & 1 & 1 & 1 & 1 & 1 & 1 & 1 & 1 & 1 & 1 \\
\hline-0.5 & 1 & 1 & 1 & 1 & 1 & 1 & 1 & 1 & 1 & 1 & 1 & 1 \\
\hline-0.4 & 1 & 1 & 1 & 1 & 1 & 1 & 1 & 1 & 1 & 1 & 1 & 1 \\
\hline-0.3 & 1 & 1 & 1 & 1 & 1 & 1 & 1 & 1 & 1 & 1 & 1 & 1 \\
\hline-0.2 & 1 & 1 & 0 & 1 & 1 & 1 & 1 & 1 & 1 & 1 & 1 & 1 \\
\hline-0.1 & 1 & 1 & 0 & 0 & 0 & 0 & 1 & 1 & 0 & 1 & 0 & 1 \\
\hline 0.0 & 0 & 0 & 0 & 0 & 0 & 0 & 0 & 0 & 0 & 0 & 0 & 0 \\
\hline 0.1 & 0 & 0 & 0 & 0 & 0 & 0 & 0 & 0 & 0 & 1 & 0 & 1 \\
\hline 0.2 & 1 & 0 & 0 & 1 & 0 & 1 & 1 & 1 & 1 & 1 & 1 & 1 \\
\hline 0.3 & 1 & 1 & 1 & 1 & 1 & 1 & 1 & 1 & 1 & 1 & 1 & 1 \\
\hline 0.4 & 0 & 1 & 1 & 1 & 1 & 1 & 1 & 1 & 1 & 1 & 1 & 1 \\
\hline 0.5 & 1 & 1 & 1 & 1 & 1 & 1 & 1 & 1 & 1 & 1 & 1 & 1 \\
\hline 0.6 & 1 & 1 & 1 & 1 & 1 & 1 & 1 & 1 & 1 & 1 & 1 & 1 \\
\hline 0.7 & 1 & 1 & 1 & 1 & 1 & 1 & 1 & 1 & 1 & 1 & 1 & 1 \\
\hline 0.8 & 1 & 1 & 1 & 1 & 1 & 1 & 1 & 1 & 1 & 1 & 1 & 1 \\
\hline
\end{tabular}


Table 4.b: Selected $k$ for $p=1$.

\begin{tabular}{|c|c|c|c|c|c|c|c|c|c|c|c|c|}
\hline & \multicolumn{12}{|c|}{ MA case: $v=(1+\theta L) e$} \\
\hline $\mathrm{T}$ & \multicolumn{6}{|c|}{100} & \multicolumn{6}{|c|}{250} \\
\hline$\theta$ & $k^{*}$ & $k^{*}$ & $k$ & $k$ & $k$ & $k$ & $k^{*}$ & $k^{*}$ & $k$ & $k$ & $k$ & $k$ \\
\hline-0.8 & 6 & 10 & 0 & 2 & 6 & 6 & 7 & 10 & 3 & 5 & 7 & 8 \\
\hline-0.7 & 4 & 8 & 1 & 2 & 4 & 5 & 5 & 8 & 3 & 4 & 5 & 6 \\
\hline-0.6 & 4 & 6 & 1 & 2 & 3 & 4 & 4 & 6 & 2 & 3 & 4 & 4 \\
\hline-0.5 & 3 & 6 & 1 & 2 & 2 & 3 & 3 & 4 & 2 & 3 & 3 & 3 \\
\hline-0.4 & 2 & 3 & 1 & 1 & 2 & 2 & 2 & 3 & 1 & 2 & 2 & 2 \\
\hline-0.3 & 2 & 2 & 0 & 1 & 1 & 1 & 2 & 2 & 1 & 1 & 1 & 2 \\
\hline-0.2 & 1 & 4 & 0 & 1 & 1 & 1 & 1 & 1 & 1 & 1 & 1 & 1 \\
\hline-0.1 & 0 & 1 & 0 & 0 & 0 & 1 & 1 & 1 & 0 & 1 & 0 & 1 \\
\hline 0.0 & 0 & 0 & 0 & 0 & 0 & 0 & 0 & 0 & 0 & 0 & 0 & 0 \\
\hline 0.1 & 1 & 0 & 0 & 1 & 0 & 0 & 1 & 0 & 0 & 1 & 0 & 1 \\
\hline 0.2 & 1 & 2 & 0 & 1 & 0 & 1 & 1 & 1 & 1 & 1 & 0 & 1 \\
\hline 0.3 & 0 & 2 & 1 & 1 & 0 & 1 & 2 & 2 & 1 & 2 & 1 & 2 \\
\hline 0.4 & 2 & 2 & 1 & 2 & 1 & 2 & 2 & 2 & 1 & 2 & 2 & 2 \\
\hline 0.5 & 2 & 2 & 1 & 2 & 2 & 2 & 2 & 2 & 2 & 3 & 2 & 3 \\
\hline 0.6 & 2 & 2 & 2 & 3 & 2 & 2 & 2 & 2 & 3 & 4 & 2 & 4 \\
\hline 0.7 & 2 & 2 & 2 & 4 & 2 & 4 & 2 & 2 & 3 & 5 & 3 & 5 \\
\hline \multirow[t]{3}{*}{0.8} & 2 & 2 & 3 & 5 & 2 & 4 & 4 & 4 & 4 & 7 & 4 & 6 \\
\hline & \multicolumn{12}{|c|}{ AR case: $(1-\rho L) v=e$} \\
\hline & \multicolumn{6}{|c|}{100} & \multicolumn{6}{|c|}{250} \\
\hline$\rho$ & $k^{*}$ & $k^{*}$ & $k$ & $k$ & $k$ & $k$ & $k^{*}$ & $k^{*}$ & $k$ & $k$ & $k$ & $k$ \\
\hline-0.8 & 3 & 1 & 1 & 1 & 1 & 1 & 4 & 1 & 1 & 1 & 1 & 1 \\
\hline-0.7 & 3 & 1 & 1 & 1 & 1 & 1 & 1 & 1 & 1 & 1 & 1 & 1 \\
\hline-0.6 & 2 & 1 & 1 & 1 & 1 & 1 & 1 & 1 & 1 & 1 & 1 & 1 \\
\hline-0.5 & 1 & 1 & 1 & 1 & 1 & 1 & 1 & 1 & 1 & 1 & 1 & 1 \\
\hline-0.4 & 1 & 1 & 1 & 1 & 1 & 1 & 1 & 1 & 1 & 1 & 1 & 1 \\
\hline-0.3 & 1 & 1 & 1 & 1 & 1 & 1 & 1 & 1 & 1 & 1 & 1 & 1 \\
\hline-0.2 & 1 & 1 & 0 & 1 & 1 & 1 & 1 & 1 & 1 & 1 & 1 & 1 \\
\hline-0.1 & 0 & 1 & 0 & 0 & 0 & 0 & 0 & 1 & 0 & 1 & 0 & 1 \\
\hline 0.0 & 0 & 0 & 0 & 0 & 0 & 0 & 0 & 0 & 0 & 0 & 0 & 0 \\
\hline 0.1 & 1 & 0 & 0 & 1 & 0 & 0 & 1 & 0 & 0 & 1 & 0 & 1 \\
\hline 0.2 & 1 & 1 & 1 & 1 & 0 & 1 & 1 & 1 & 1 & 1 & 0 & 1 \\
\hline 0.3 & 1 & 1 & 1 & 1 & 0 & 1 & 1 & 1 & 1 & 1 & 1 & 1 \\
\hline 0.4 & 1 & 1 & 1 & 1 & 1 & 1 & 1 & 1 & 1 & 1 & 1 & 1 \\
\hline 0.5 & 1 & 1 & 1 & 1 & 1 & 1 & 1 & 1 & 1 & 1 & 1 & 1 \\
\hline 0.6 & 1 & 1 & 1 & 1 & 1 & 1 & 1 & 1 & 1 & 1 & 1 & 1 \\
\hline 0.7 & 1 & 1 & 1 & 1 & 1 & 1 & 1 & 1 & 1 & 1 & 1 & 1 \\
\hline 0.8 & 1 & 1 & 1 & 1 & 1 & 1 & 1 & 1 & 1 & 1 & 1 & 1 \\
\hline
\end{tabular}

$\mathrm{a} \quad \mathrm{m} \quad \mathrm{a} \quad \mathrm{a} \quad k \quad 5000 \quad \mathrm{a}$ 
Table 5.a: Size of the Tests $p=0$.

MA case: $v=e+\theta e_{-1}, e \sim N(0,1)$

\begin{tabular}{|c|c|ccc|cccc|ccc|}
\hline \hline & & & & & & & & & & & \\
$T$ & $\theta$ & $M$ & $M^{G L S}$ & $\bar{M}^{G L S}$ & $D F$ & $D F^{G L S}$ & $D F^{G L S}$ & $D F^{G L S}$ & $P_{T}$ & $\bar{P}_{T}^{G L S}$ & $\bar{M} P_{T}^{G L S}$ \\
\hline 100 & -0.8 & 0.058 & 0.041 & 0.036 & 0.394 & 0.411 & 0.356 & 0.107 & 0.429 & 0.034 & 0.036 \\
& -0.5 & 0.028 & 0.013 & 0.056 & 0.082 & 0.104 & 0.095 & 0.073 & 0.109 & 0.048 & 0.051 \\
& 0.0 & 0.018 & 0.005 & 0.043 & 0.072 & 0.077 & 0.072 & 0.050 & 0.094 & 0.036 & 0.040 \\
& 0.5 & 0.027 & 0.013 & 0.062 & 0.081 & 0.082 & 0.080 & 0.048 & 0.118 & 0.048 & 0.054 \\
& 0.8 & 0.042 & 0.018 & 0.067 & 0.095 & 0.097 & 0.091 & 0.039 & 0.145 & 0.054 & 0.062 \\
\hline 150 & -0.8 & 0.034 & 0.018 & 0.026 & 0.407 & 0.390 & 0.304 & 0.095 & 0.420 & 0.025 & 0.027 \\
& -0.5 & 0.025 & 0.009 & 0.054 & 0.085 & 0.093 & 0.086 & 0.066 & 0.091 & 0.047 & 0.049 \\
& 0.0 & 0.017 & 0.005 & 0.049 & 0.062 & 0.075 & 0.070 & 0.051 & 0.085 & 0.040 & 0.046 \\
& 0.5 & 0.028 & 0.011 & 0.054 & 0.066 & 0.072 & 0.071 & 0.046 & 0.090 & 0.047 & 0.050 \\
& 0.8 & 0.040 & 0.013 & 0.060 & 0.079 & 0.077 & 0.070 & 0.034 & 0.108 & 0.047 & 0.052 \\
\hline 250 & -0.8 & 0.016 & 0.006 & 0.022 & 0.370 & 0.323 & 0.236 & 0.088 & 0.380 & 0.021 & 0.021 \\
& -0.5 & 0.036 & 0.012 & 0.058 & 0.075 & 0.089 & 0.079 & 0.063 & 0.086 & 0.049 & 0.054 \\
& 0.0 & 0.021 & 0.006 & 0.047 & 0.059 & 0.062 & 0.062 & 0.045 & 0.069 & 0.039 & 0.042 \\
& 0.5 & 0.034 & 0.009 & 0.056 & 0.059 & 0.068 & 0.069 & 0.045 & 0.078 & 0.047 & 0.051 \\
& 0.8 & 0.044 & 0.014 & 0.059 & 0.066 & 0.062 & 0.058 & 0.042 & 0.081 & 0.050 & 0.053 \\
\hline
\end{tabular}

AR case: $v=\rho v_{-1}+e, e \sim N(0,1)$

\begin{tabular}{|c|c|ccc|cccc|ccc|}
\hline \hline & & & & & & & & & & & \\
$T$ & $\rho$ & $M$ & $M^{G L S}$ & $\bar{M}^{G L S}$ & $D F$ & $D F^{G L S}$ & $D F^{G L S}$ & $D F^{G L S}$ & $P_{T}$ & $\bar{P}_{T}^{G L S}$ & $\bar{M} P_{T}^{G L S}$ \\
\hline 100 & -0.8 & 0.002 & 0.000 & 0.015 & 0.067 & 0.082 & 0.076 & 0.049 & 0.063 & 0.013 & 0.014 \\
& -0.5 & 0.010 & 0.003 & 0.037 & 0.067 & 0.076 & 0.071 & 0.046 & 0.087 & 0.028 & 0.033 \\
& 0.5 & 0.029 & 0.012 & 0.064 & 0.082 & 0.087 & 0.082 & 0.053 & 0.108 & 0.051 & 0.057 \\
& 0.8 & 0.047 & 0.025 & 0.090 & 0.091 & 0.081 & 0.077 & 0.053 & 0.125 & 0.068 & 0.077 \\
\hline 150 & -0.8 & 0.003 & 0.000 & 0.021 & 0.062 & 0.069 & 0.067 & 0.048 & 0.051 & 0.019 & 0.020 \\
& -0.5 & 0.013 & 0.004 & 0.039 & 0.060 & 0.074 & 0.069 & 0.045 & 0.072 & 0.031 & 0.035 \\
& 0.5 & 0.030 & 0.010 & 0.055 & 0.063 & 0.069 & 0.066 & 0.047 & 0.081 & 0.046 & 0.051 \\
& 0.8 & 0.043 & 0.017 & 0.070 & 0.067 & 0.071 & 0.069 & 0.047 & 0.092 & 0.055 & 0.063 \\
\hline 250 & -0.8 & 0.007 & 0.001 & 0.029 & 0.056 & 0.057 & 0.054 & 0.040 & 0.049 & 0.026 & 0.028 \\
& -0.5 & 0.022 & 0.006 & 0.046 & 0.052 & 0.057 & 0.058 & 0.047 & 0.062 & 0.040 & 0.042 \\
& 0.5 & 0.028 & 0.008 & 0.052 & 0.056 & 0.061 & 0.061 & 0.045 & 0.070 & 0.043 & 0.046 \\
& 0.8 & 0.037 & 0.010 & 0.060 & 0.060 & 0.061 & 0.063 & 0.051 & 0.073 & 0.051 & 0.056 \\
\hline
\end{tabular}

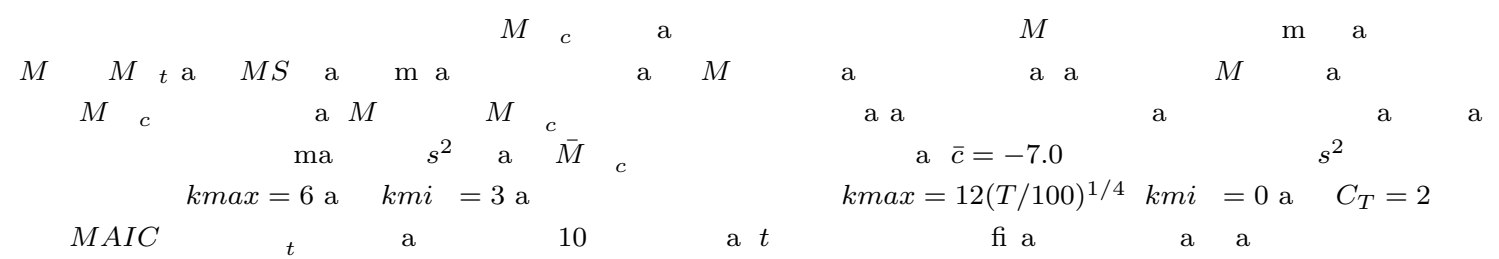


Table 5.b: Size Ad usted Power of the Tests $p=0$.

MA case: $v=e+\theta e_{-1}, e \sim N(0,1)$

\begin{tabular}{|c|c|ccc|cccc|ccc|}
\hline \hline & & & & & & & & & & & \\
$T$ & $\theta$ & $M$ & $M^{G L S}$ & $\bar{M}^{G L S}$ & $D F$ & $D F^{G L S}$ & $D F^{G L S}$ & $D F^{G L S}$ & $P_{T}$ & $\bar{P}_{T}^{G L S}$ & $\bar{M} P_{T}^{G L S}$ \\
\hline 100 & -0.8 & 0.193 & 0.237 & 0.311 & 0.241 & 0.419 & 0.412 & 0.385 & 0.313 & 0.326 & 0.311 \\
& -0.5 & 0.253 & 0.351 & 0.344 & 0.191 & 0.448 & 0.429 & 0.385 & 0.414 & 0.362 & 0.355 \\
& 0.0 & 0.315 & 0.452 & 0.458 & 0.131 & 0.432 & 0.436 & 0.454 & 0.418 & 0.471 & 0.442 \\
& 0.5 & 0.259 & 0.332 & 0.324 & 0.122 & 0.414 & 0.402 & 0.370 & 0.402 & 0.351 & 0.347 \\
& 0.8 & 0.180 & 0.257 & 0.264 & 0.134 & 0.366 & 0.335 & 0.343 & 0.348 & 0.296 & 0.281 \\
\hline 150 & -0.8 & 0.180 & 0.252 & 0.296 & 0.262 & 0.448 & 0.417 & 0.415 & 0.381 & 0.307 & 0.292 \\
& -0.5 & 0.267 & 0.387 & 0.384 & 0.195 & 0.450 & 0.430 & 0.393 & 0.421 & 0.398 & 0.396 \\
& 0.0 & 0.339 & 0.463 & 0.460 & 0.152 & 0.422 & 0.405 & 0.454 & 0.413 & 0.467 & 0.453 \\
& 0.5 & 0.263 & 0.382 & 0.380 & 0.160 & 0.441 & 0.418 & 0.405 & 0.429 & 0.393 & 0.398 \\
& 0.8 & 0.233 & 0.346 & 0.363 & 0.147 & 0.382 & 0.362 & 0.404 & 0.387 & 0.377 & 0.374 \\
\hline 250 & -0.8 & 0.190 & 0.319 & 0.334 & 0.287 & 0.441 & 0.420 & 0.437 & 0.419 & 0.335 & 0.327 \\
& -0.5 & 0.264 & 0.404 & 0.396 & 0.201 & 0.477 & 0.455 & 0.428 & 0.458 & 0.425 & 0.409 \\
& 0.0 & 0.338 & 0.481 & 0.477 & 0.152 & 0.464 & 0.446 & 0.483 & 0.455 & 0.485 & 0.478 \\
& 0.5 & 0.258 & 0.411 & 0.411 & 0.169 & 0.439 & 0.424 & 0.431 & 0.441 & 0.422 & 0.413 \\
& 0.8 & 0.246 & 0.385 & 0.395 & 0.143 & 0.432 & 0.413 & 0.419 & 0.434 & 0.406 & 0.405 \\
\hline
\end{tabular}

AR case: $v=\rho v_{-1}+e, e \sim N(0,1)$

\begin{tabular}{|c|c|ccc|cccc|ccc|}
\hline \hline & & & & & & & & & & & \\
$T$ & $\rho$ & $M$ & $M^{G L S}$ & $\bar{M}^{G L S}$ & $D F$ & $D F^{G L S}$ & $D F^{G L S}$ & $D F^{G L S}$ & $P_{T}$ & $\bar{P}_{T}^{G L S}$ & $\bar{M} P_{T}^{G L S}$ \\
\hline 100 & -0.8 & 0.246 & 0.349 & 0.350 & 0.154 & 0.433 & 0.429 & 0.404 & 0.384 & 0.362 & 0.342 \\
& -0.5 & 0.330 & 0.437 & 0.432 & 0.145 & 0.428 & 0.420 & 0.455 & 0.405 & 0.452 & 0.437 \\
& 0.5 & 0.279 & 0.365 & 0.368 & 0.115 & 0.338 & 0.330 & 0.385 & 0.379 & 0.385 & 0.374 \\
& 0.8 & 0.196 & 0.251 & 0.241 & 0.084 & 0.297 & 0.292 & 0.298 & 0.261 & 0.276 & 0.269 \\
\hline 150 & -0.8 & 0.275 & 0.398 & 0.416 & 0.161 & 0.477 & 0.437 & 0.432 & 0.442 & 0.419 & 0.409 \\
& -0.5 & 0.332 & 0.453 & 0.454 & 0.161 & 0.404 & 0.390 & 0.468 & 0.423 & 0.463 & 0.458 \\
& 0.5 & 0.307 & 0.419 & 0.411 & 0.140 & 0.417 & 0.410 & 0.446 & 0.407 & 0.436 & 0.430 \\
& 0.8 & 0.232 & 0.333 & 0.330 & 0.103 & 0.341 & 0.324 & 0.362 & 0.325 & 0.349 & 0.353 \\
\hline 250 & -0.8 & 0.325 & 0.479 & 0.472 & 0.157 & 0.485 & 0.480 & 0.493 & 0.468 & 0.477 & 0.471 \\
& -0.5 & 0.300 & 0.452 & 0.447 & 0.179 & 0.493 & 0.477 & 0.466 & 0.469 & 0.459 & 0.460 \\
& 0.5 & 0.336 & 0.443 & 0.436 & 0.158 & 0.447 & 0.434 & 0.456 & 0.428 & 0.468 & 0.456 \\
& 0.8 & 0.279 & 0.375 & 0.366 & 0.131 & 0.386 & 0.382 & 0.381 & 0.381 & 0.387 & 0.383 \\
\hline
\end{tabular}

$T=250$

a a $\quad$ a $\quad \bar{\alpha}=1+\bar{c} / T \quad \bar{c}=-7.0 \quad$ a $\quad$ a $\quad 0.3 \quad 0.53$ a $\quad 0.72 \quad T=100 \quad T=150$ a 
Table 6.a: Size of the Tests $p=1$.

MA case: $v=e+\theta e_{-1}, e \sim N(0,1)$

\begin{tabular}{|c|c|ccc|cccc|ccc|}
\hline \hline & & & & & & & & & & & \\
$T$ & $\theta$ & $M$ & $M^{G L S}$ & $\bar{M}^{G L S}$ & $D F$ & $D F^{G L S}$ & $D F^{G L S}$ & $D F^{G L S}$ & $P_{T}$ & $\bar{P}_{T}^{G L S}$ & $\bar{M} P_{T}^{G L S}$ \\
\hline 100 & -0.8 & 0.140 & 0.123 & 0.059 & 0.509 & 0.567 & 0.528 & 0.123 & 0.562 & 0.061 & 0.061 \\
& -0.5 & 0.037 & 0.025 & 0.035 & 0.092 & 0.107 & 0.111 & 0.069 & 0.145 & 0.036 & 0.036 \\
& 0.0 & 0.011 & 0.005 & 0.018 & 0.075 & 0.074 & 0.078 & 0.039 & 0.161 & 0.017 & 0.018 \\
& 0.5 & 0.032 & 0.022 & 0.048 & 0.084 & 0.085 & 0.094 & 0.038 & 0.190 & 0.046 & 0.047 \\
& 0.8 & 0.048 & 0.033 & 0.056 & 0.096 & 0.105 & 0.109 & 0.017 & 0.250 & 0.053 & 0.056 \\
\hline 150 & -0.8 & 0.074 & 0.061 & 0.027 & 0.581 & 0.586 & 0.477 & 0.082 & 0.570 & 0.028 & 0.028 \\
& -0.5 & 0.032 & 0.017 & 0.028 & 0.087 & 0.103 & 0.104 & 0.049 & 0.114 & 0.028 & 0.029 \\
& 0.0 & 0.017 & 0.008 & 0.023 & 0.066 & 0.067 & 0.072 & 0.038 & 0.111 & 0.023 & 0.024 \\
& 0.5 & 0.025 & 0.016 & 0.032 & 0.068 & 0.084 & 0.085 & 0.029 & 0.142 & 0.032 & 0.034 \\
& 0.8 & 0.058 & 0.035 & 0.058 & 0.080 & 0.088 & 0.085 & 0.018 & 0.181 & 0.055 & 0.057 \\
\hline 250 & -0.8 & 0.025 & 0.016 & 0.012 & 0.566 & 0.531 & 0.381 & 0.064 & 0.554 & 0.012 & 0.012 \\
& -0.5 & 0.031 & 0.016 & 0.033 & 0.094 & 0.105 & 0.099 & 0.048 & 0.103 & 0.034 & 0.035 \\
& 0.0 & 0.023 & 0.011 & 0.029 & 0.056 & 0.059 & 0.065 & 0.038 & 0.083 & 0.030 & 0.031 \\
& 0.5 & 0.032 & 0.012 & 0.037 & 0.062 & 0.066 & 0.070 & 0.028 & 0.097 & 0.036 & 0.037 \\
& 0.8 & 0.059 & 0.032 & 0.059 & 0.062 & 0.067 & 0.060 & 0.026 & 0.119 & 0.059 & 0.060 \\
\hline
\end{tabular}

AR case: $v=\rho v_{-1}+e, e \sim N(0,1)$

\begin{tabular}{|c|c|ccc|cccc|ccc|}
\hline \hline \multirow{2}{*}{$T$} & $\rho$ & $M$ & $M^{G L S}$ & $\bar{M}^{G L S}$ & $D F$ & $D F^{G L S}$ & $D F^{G L S}$ & $D F^{G L S}$ & $P_{T}$ & $\bar{P}_{T}^{G L S}$ & $\bar{M} P_{T}^{G L S}$ \\
\hline 100 & -0.8 & 0.000 & 0.000 & 0.000 & 0.072 & 0.079 & 0.084 & 0.034 & 0.067 & 0.001 & 0.000 \\
& -0.5 & 0.006 & 0.003 & 0.012 & 0.071 & 0.074 & 0.077 & 0.037 & 0.122 & 0.013 & 0.013 \\
& 0.5 & 0.033 & 0.021 & 0.042 & 0.076 & 0.083 & 0.088 & 0.038 & 0.178 & 0.040 & 0.043 \\
& 0.8 & 0.081 & 0.056 & 0.093 & 0.081 & 0.086 & 0.091 & 0.043 & 0.210 & 0.080 & 0.085 \\
\hline 150 & -0.8 & 0.001 & 0.000 & 0.003 & 0.061 & 0.062 & 0.069 & 0.028 & 0.043 & 0.001 & 0.004 \\
& -0.5 & 0.017 & 0.006 & 0.024 & 0.059 & 0.070 & 0.075 & 0.038 & 0.090 & 0.014 & 0.023 \\
& 0.5 & 0.037 & 0.018 & 0.044 & 0.056 & 0.070 & 0.074 & 0.039 & 0.127 & 0.041 & 0.043 \\
& 0.8 & 0.045 & 0.022 & 0.056 & 0.063 & 0.066 & 0.073 & 0.043 & 0.142 & 0.056 & 0.055 \\
\hline 250 & -0.8 & 0.001 & 0.000 & 0.003 & 0.057 & 0.054 & 0.058 & 0.028 & 0.009 & 0.004 & 0.004 \\
& -0.5 & 0.017 & 0.006 & 0.024 & 0.046 & 0.047 & 0.053 & 0.038 & 0.025 & 0.023 & 0.023 \\
& 0.5 & 0.037 & 0.018 & 0.044 & 0.059 & 0.063 & 0.068 & 0.039 & 0.046 & 0.042 & 0.043 \\
& 0.8 & 0.045 & 0.022 & 0.056 & 0.054 & 0.056 & 0.061 & 0.043 & 0.054 & 0.052 & 0.055 \\
\hline
\end{tabular}

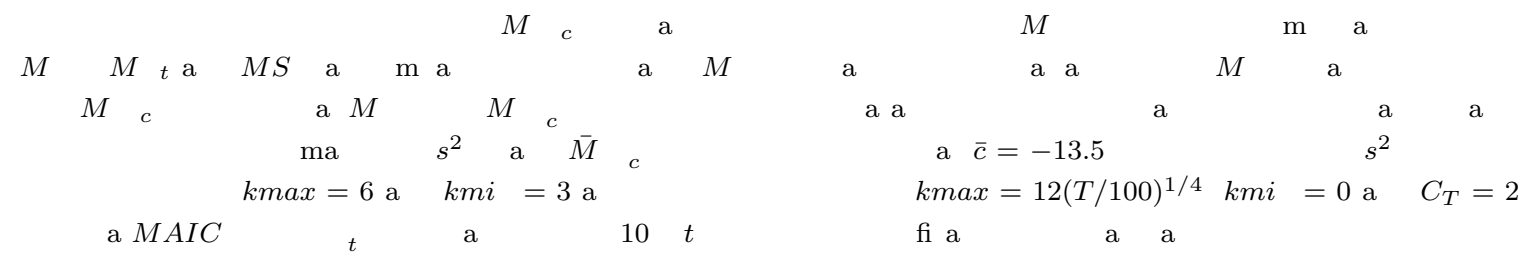


Table 6.b: Size Ad usted Power of the Tests $p=1$.

MA case: $v=e+\theta e_{-1}, e \sim N(0,1)$

\begin{tabular}{|c|c|ccc|cccc|ccc|}
\hline \hline & & & & & & & & & & & \\
$T$ & $\theta$ & $M$ & $M^{G L S}$ & $\bar{M}^{G L S}$ & $D F$ & $D F^{G L S}$ & $D F^{G L S}$ & $D F^{G L S}$ & $P_{T}$ & $\bar{P}_{T}^{G L S}$ & $\bar{M} P_{T}^{G L S}$ \\
\hline 100 & -0.8 & 0.296 & 0.266 & 0.348 & 0.283 & 0.398 & 0.414 & 0.357 & 0.355 & 0.350 & 0.348 \\
& -0.5 & 0.320 & 0.246 & 0.300 & 0.258 & 0.421 & 0.420 & 0.324 & 0.324 & 0.311 & 0.305 \\
& 0.0 & 0.450 & 0.266 & 0.439 & 0.175 & 0.362 & 0.341 & 0.432 & 0.488 & 0.448 & 0.442 \\
& 0.5 & 0.196 & 0.147 & 0.207 & 0.161 & 0.316 & 0.310 & 0.259 & 0.320 & 0.224 & 0.218 \\
& 0.8 & 0.149 & 0.166 & 0.154 & 0.169 & 0.282 & 0.269 & 0.254 & 0.260 & 0.163 & 0.155 \\
\hline 150 & -0.8 & 0.236 & 0.269 & 0.316 & 0.309 & 0.444 & 0.416 & 0.354 & 0.326 & 0.316 & 0.314 \\
& -0.5 & 0.283 & 0.348 & 0.325 & 0.291 & 0.434 & 0.415 & 0.358 & 0.384 & 0.333 & 0.330 \\
& 0.0 & 0.407 & 0.470 & 0.457 & 0.200 & 0.384 & 0.352 & 0.462 & 0.369 & 0.466 & 0.465 \\
& 0.5 & 0.312 & 0.347 & 0.348 & 0.212 & 0.365 & 0.365 & 0.375 & 0.354 & 0.344 & 0.348 \\
& 0.8 & 0.171 & 0.209 & 0.217 & 0.201 & 0.317 & 0.310 & 0.309 & 0.302 & 0.227 & 0.225 \\
\hline 250 & -0.8 & 0.247 & 0.285 & 0.296 & 0.371 & 0.460 & 0.430 & 0.375 & 0.406 & 0.300 & 0.297 \\
& -0.5 & 0.308 & 0.370 & 0.366 & 0.278 & 0.459 & 0.441 & 0.392 & 0.422 & 0.374 & 0.371 \\
& 0.0 & 0.380 & 0.458 & 0.451 & 0.250 & 0.437 & 0.418 & 0.447 & 0.432 & 0.455 & 0.456 \\
& 0.5 & 0.311 & 0.374 & 0.365 & 0.258 & 0.426 & 0.406 & 0.388 & 0.441 & 0.367 & 0.363 \\
& 0.8 & 0.225 & 0.268 & 0.271 & 0.233 & 0.364 & 0.354 & 0.310 & 0.388 & 0.280 & 0.282 \\
\hline
\end{tabular}

AR case: $v=\rho v_{-1}+e, e \sim N(0,1)$

\begin{tabular}{|c|c|ccc|cccc|ccc|}
\hline \hline & & & & & & & & & & & \\
$T$ & $\rho$ & $M$ & $M^{G L S}$ & $\bar{M}^{G L S}$ & $D F$ & $D F^{G L S}$ & $D F^{G L S}$ & $D F^{G L S}$ & $P_{T}$ & $\bar{P}_{T}^{G L S}$ & $\bar{M} P_{T}^{G L S}$ \\
\hline 100 & -0.8 & 0.257 & 0.308 & 0.296 & 0.204 & 0.380 & 0.372 & 0.385 & 0.256 & 0.301 & 0.299 \\
& -0.5 & 0.332 & 0.396 & 0.382 & 0.205 & 0.367 & 0.366 & 0.431 & 0.303 & 0.398 & 0.393 \\
& 0.5 & 0.235 & 0.265 & 0.270 & 0.133 & 0.262 & 0.264 & 0.286 & 0.247 & 0.279 & 0.280 \\
& 0.8 & 0.114 & 0.127 & 0.129 & 0.098 & 0.167 & 0.165 & 0.206 & 0.144 & 0.150 & 0.144 \\
\hline 150 & -0.8 & 0.311 & 0.363 & 0.349 & 0.240 & 0.435 & 0.409 & 0.409 & 0.345 & 0.351 & 0.347 \\
& -0.5 & 0.388 & 0.433 & 0.423 & 0.240 & 0.404 & 0.376 & 0.437 & 0.385 & 0.438 & 0.429 \\
& 0.5 & 0.296 & 0.338 & 0.345 & 0.207 & 0.338 & 0.313 & 0.366 & 0.333 & 0.358 & 0.355 \\
& 0.8 & 0.189 & 0.211 & 0.218 & 0.133 & 0.239 & 0.228 & 0.251 & 0.211 & 0.228 & 0.224 \\
\hline 250 & -0.8 & 0.379 & 0.452 & 0.441 & 0.245 & 0.472 & 0.456 & 0.468 & 0.464 & 0.446 & 0.442 \\
& -0.5 & 0.372 & 0.453 & 0.451 & 0.300 & 0.509 & 0.484 & 0.445 & 0.445 & 0.452 & 0.457 \\
& 0.5 & 0.331 & 0.387 & 0.385 & 0.233 & 0.391 & 0.372 & 0.392 & 0.401 & 0.395 & 0.388 \\
& 0.8 & 0.259 & 0.280 & 0.288 & 0.189 & 0.314 & 0.292 & 0.289 & 0.312 & 0.285 & 0.284 \\
\hline
\end{tabular}

$T=250$

a a $\quad$ a $\quad \bar{\alpha}=1+\bar{c} / T \quad \bar{c}=-13.5 \quad$ a $\quad$ a $\quad 0.865 \quad 0.10$ a $\quad 0.46 \quad T=100 \quad T=150$ a 
Table 7: Empirical Results for Inflation Series from the G7 countries.

\begin{tabular}{|c|c|ccccccc|}
\hline \hline & & USA & Canada & U.K. & Japan & Italy & France & Germany \\
\hline & $A R(\alpha)$ & .95 & .95 & .93 & .87 & .92 & .98 & .92 \\
& $M A(\theta)$ & -.32 & -.51 & -.55 & -.42 & -.27 & -.72 & -.54 \\
\hline BIC & $k$ & 2 & 1 & 1 & 1 & 0 & 3 & 3 \\
& $\bar{Z}_{\alpha}^{G L S}$ & -4.04 & -9.63 & -26.81 & -34.12 & -7.39 & -5.70 & -26.58 \\
& $\bar{M} Z_{\alpha}^{G L S}$ & -3.71 & -8.13 & -18.05 & -23.95 & -7.17 & -3.49 & -16.57 \\
& $\bar{D} F^{G L S}$ & -1.37 & -2.07 & -3.31 & -3.85 & -1.95 & -1.36 & -3.00 \\
& $\bar{P}_{T}^{G L S}$ & 7.39 & 3.36 & 1.40 & 1.01 & 4.06 & 7.92 & 1.96 \\
& $\bar{M} P_{T}^{G L S}$ & 6.60 & 3.12 & 1.41 & 1.02 & 3.42 & 7.01 & 1.98 \\
\hline MAIC & $k$ & 2 & 2 & 8 & 12 & 5 & 7 & 5 \\
& $\bar{Z}_{\alpha}^{G L S}$ & -4.04 & -6.98 & -14.75 & -13.35 & -2.71 & -3.27 & -18.83 \\
& $\bar{M} Z_{\alpha}^{G L S}$ & -3.71 & -5.48 & -6.00 & -3.17 & -2.49 & -1.07 & -8.82 \\
& $\bar{D} F G L S$ & -1.37 & -1.69 & 1.77 & -1.33 & -1.08 & -0.77 & -2.21 \\
& $P_{T}^{G L S}$ & 7.39 & 4.92 & 4.13 & 7.64 & 11.68 & 25.85 & 3.42 \\
& $\bar{M} P_{T}^{G L S}$ & 6.60 & 4.58 & 4.17 & 7.73 & 9.83 & 22.89 & 3.44 \\
\hline \hline
\end{tabular}

Note: $a, b$ and $c$ denotes a statistic significant at the $1 \%, 5 \%$ and $10 \%$ level, respectively. 
Table 3.a: alues of of the proportionalinity factor $\kappa$

\begin{tabular}{|c|c|c|c|c|c|c|c|c|c|c|c|c|c|c|c|c|c|c|c|c|c|}
\hline & \multicolumn{4}{|c|}{$=100$} & \multicolumn{4}{|c|}{$=150$} & \multicolumn{4}{|c|}{$=250$} & \multicolumn{4}{|c|}{$=500$} & \multicolumn{4}{|c|}{$=5000$} & \multirow[b]{2}{*}{ bic } \\
\hline$\delta$ & bic & aic & mbic & maic & bic & aic & mbic & maic & bic & aic & mbic & maic & bic & aic & mbic & maic & bic & aic & mbic & maic & \\
\hline \multicolumn{22}{|c|}{$p=0$} \\
\hline 5 & & 6 & 35 & 170 & & 6 & 358 & 163 & & 6 & 355 & 155 & & 6 & 347 & 144 & & 6 & 306 & 114 & \\
\hline 10 & 6 & 65 & 173 & 88 & 6 & 65 & 173 & 85 & 7 & 65 & 171 & 82 & 7 & 65 & 168 & 7 & 8 & 65 & 152 & 71 & 8 \\
\hline 15 & 3 & 61 & 124 & 68 & 3 & 61 & 124 & 67 & 4 & 61 & 124 & 66 & 4 & 61 & 123 & 65 & 6 & 61 & 116 & 62 & 6 \\
\hline 20 & 8 & 57 & 104 & 5 & 0 & 57 & 104 & 5 & 0 & 57 & 104 & 58 & 1 & 57 & 104 & 58 & 3 & 57 & 102 & 57 & 4 \\
\hline 50 & 67 & 3 & 68 & 3 & 6 & 3 & 6 & 3 & 70 & 3 & 71 & 3 & 72 & 3 & 72 & 3 & 76 & 3 & 76 & 3 & 7 \\
\hline 80 & 54 & 30 & 54 & 30 & 55 & 30 & 55 & 30 & 57 & 30 & 57 & 30 & 58 & 30 & 58 & 30 & 63 & 30 & 63 & 30 & 66 \\
\hline \multicolumn{22}{|c|}{$p=1$} \\
\hline 5 & & 6 & 634 & $\begin{array}{ll}2 & 6\end{array}$ & & 6 & 641 & 287 & & 6 & 646 & 275 & & 6 & 646 & 260 & & 6 & 612 & 212 & \\
\hline 10 & 6 & 65 & 28 & 137 & 6 & 65 & 22 & 132 & 67 & 65 & 23 & 127 & 7 & 65 & 22 & 120 & 8 & 65 & 274 & 100 & 8 \\
\hline 15 & 3 & 61 & 187 & 0 & 3 & 61 & 188 & 88 & 4 & 61 & 18 & 85 & 4 & 61 & 18 & 81 & 6 & 61 & 178 & 72 & 6 \\
\hline 20 & 8 & 57 & 40 & 70 & 0 & 57 & 142 & 6 & 0 & 57 & 142 & 67 & 1 & 57 & 142 & 65 & 3 & 57 & 136 & 60 & 4 \\
\hline 50 & 67 & 3 & 71 & 3 & 6 & 3 & 72 & 3 & 70 & 3 & 73 & 3 & 72 & 3 & 75 & 3 & 76 & 3 & 78 & 3 & 7 \\
\hline 80 & 54 & 30 & 54 & 30 & 55 & 30 & 56 & 30 & 57 & 30 & 57 & 30 & 58 & 30 & 5 & 30 & 63 & 30 & 63 & 30 & 66 \\
\hline
\end{tabular}

Table 3.b: $k$ Chosen by inimizing the Asymptotic Approximation of the IC and IC 14

\begin{tabular}{|c|c|c|c|c|c|c|c|c|c|c|c|c|c|c|c|c|c|c|c|}
\hline & \multicolumn{4}{|c|}{$\mathrm{T}=100$} & \multicolumn{4}{|c|}{$\mathrm{T}=150$} & \multicolumn{4}{|c|}{$\mathrm{T}=250$} & \multicolumn{4}{|c|}{$\mathrm{T}=500$} & \multicolumn{3}{|c|}{$\mathrm{T}=5000$} \\
\hline$\delta$ & bic & aic & mbic & maic & bic & aic & mbic & maic & bic & aic & mbic & maic & $b i c$ & aic & $m b i c$ & maic & bic & aic & mbic \\
\hline \multicolumn{20}{|c|}{$p=0$} \\
\hline .5 & 4 & 6 & 16 & 17 & 5 & 8 & 19 & 20 & 6 & 10 & 23 & 24 & 8 & 15 & 31 & 32 & 24 & 49 & 74 \\
\hline 1.0 & 4 & 6 & 8 & 8 & 5 & 8 & 9 & 10 & 6 & 10 & 11 & 13 & 8 & 14 & 15 & 17 & 23 & 46 & 37 \\
\hline 1.5 & 4 & 6 & 5 & 6 & 5 & 7 & 6 & 8 & 6 & 9 & 8 & 10 & 8 & 13 & 11 & 14 & 23 & 43 & 28 \\
\hline 2.0 & 4 & 5 & 4 & 5 & 4 & 7 & 5 & 7 & 6 & 9 & 7 & 9 & 8 & 12 & 9 & 13 & 22 & 40 & 24 \\
\hline 5.0 & 3 & 3 & 3 & 3 & 3 & 4 & 3 & 4 & 4 & 6 & 4 & 6 & 6 & 8 & 6 & 8 & 18 & 27 & 18 \\
\hline 8.0 & 2 & 3 & 2 & 3 & 3 & 3 & 3 & 3 & 3 & 4 & 3 & 4 & 5 & 6 & 5 & 6 & 15 & 21 & 15 \\
\hline \multicolumn{20}{|c|}{$p=1$} \\
\hline .5 & 4 & 6 & 29 & 29 & 5 & 8 & 35 & 35 & 6 & 10 & 43 & 43 & 8 & 15 & 57 & 58 & 24 & 49 & 148 \\
\hline 1.0 & 4 & 6 & 13 & 13 & 5 & 8 & 15 & 16 & 6 & 10 & 19 & 20 & 8 & 14 & 26 & 27 & 23 & 46 & 66 \\
\hline 1.5 & 4 & 6 & 8 & 9 & 5 & 7 & 10 & 10 & 6 & 9 & 12 & 13 & 8 & 13 & 16 & 18 & 23 & 43 & 43 \\
\hline 2.0 & 4 & 5 & 6 & 7 & 4 & 7 & 7 & 8 & 6 & 9 & 9 & 10 & 8 & 12 & 12 & 14 & 22 & 40 & 33 \\
\hline 5.0 & 3 & 3 & 3 & 3 & 3 & 4 & 3 & 4 & 4 & 6 & 4 & 6 & 6 & 8 & 6 & 8 & 18 & 27 & 19 \\
\hline 8.0 & 2 & 3 & 2 & 3 & 3 & 3 & 3 & 3 & 3 & 4 & 3 & 4 & 5 & 6 & 5 & 6 & 15 & 21 & 15 \\
\hline
\end{tabular}


Figure $1: \tau_{T}(k)$
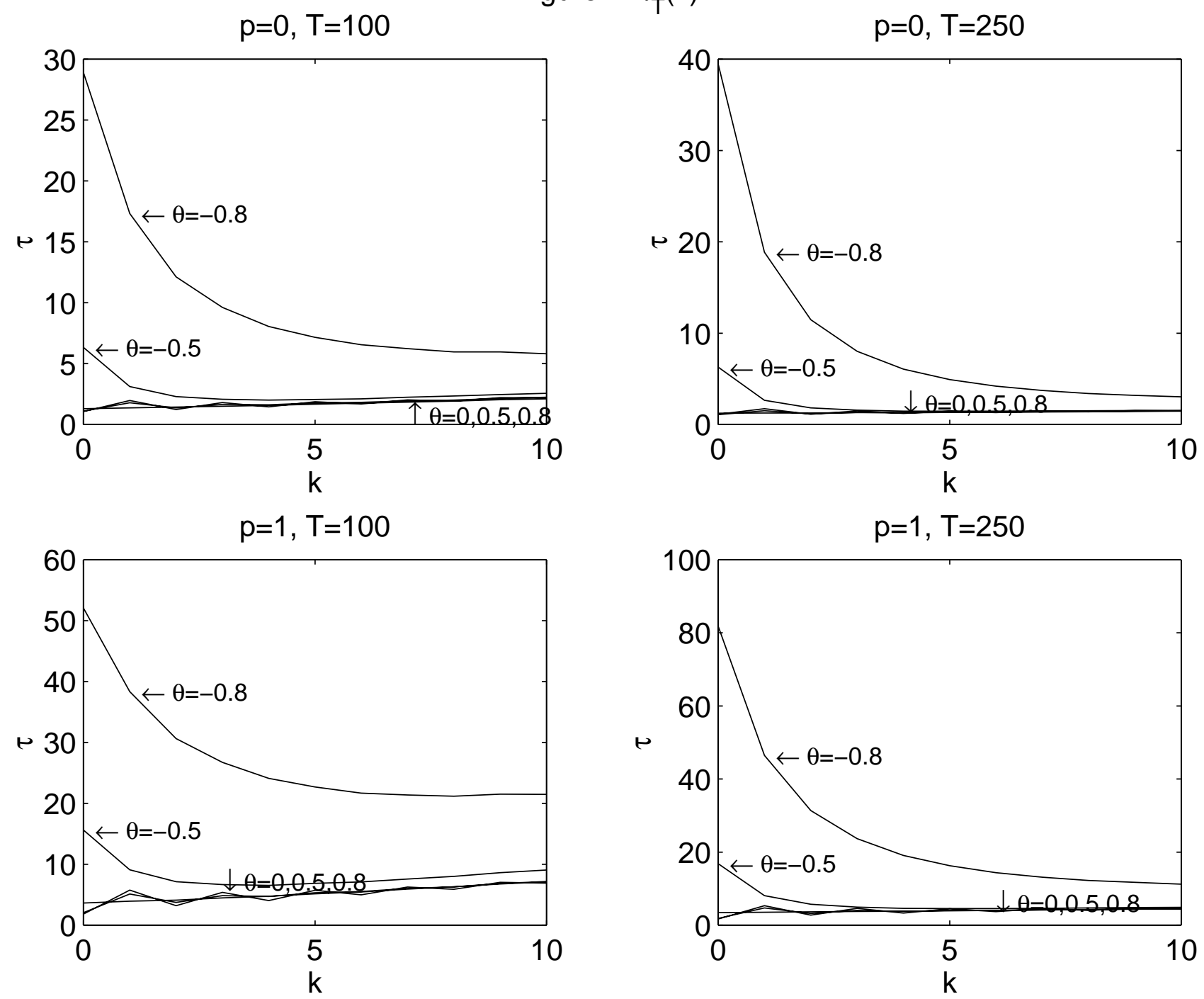